\title{
A GENERAL UNIFIED APPROACH TO MODELLING SWITCHING DC-TO-DC CONVERTERS IN DISCONTINUOUS CONDUCTION MODE
}

\author{
SLOBODAN ĆUK and R.D. MIDDLEBROOK \\ Californio Instifute of Technology \\ Pasadena, Californio
}

\section{ABSTRACT}

A method for modelling switching converters in the discontinuous conduction mode is developed, whose starting point is the unified state-space representation, and whose end result is a complete linear circuit model which correctly represents all essential features, namely, the input, output, and transfer properties (static dc as well as dynamic ac small signal). While the method is generally applicable to any switching converter operating in the discontinuous conduction mode, it is extensively illustrated for the three common power stages (buck, boost, and buck-boost). The results for these converters are then easily tabulated owing to the fixed equivalent circuit topology of their canonical circuit model.

The outlined method lends itself easily to investigation of the discontinuous conduction mode in more complex structures ( cascade connection of buck and boost converters, for examplel, in which more than one inductor current may become discontinuous.

As opposed to other modelling techniques, the new method considers the discontinuous conduction mode as a special case of the continuous conduction mode.

\section{$1 \quad$ INTRODUCTION}

Switching-mode dc-to-dc converters afford an efficient means of transforming power at one dc voltage to another. There are many circuit configurations capable of performing dc-to-dc conversion, of which the most common are the buck, boost, and buck-boost converters shown in Fig. 1. In each converter, the basic dc-to-dc conversion function is achieved by control of the switch fractional closed-time (transistor on-time), or duty ratio, $D(0<D<1)$ with constant switching frequency $f_{s} \triangleq 1 / T_{s}$, where $T_{s}$ is the switching
period.

Two modes of switching converter operation may be distinguished: the continuous conduction mode (Inductor current never falls to zero, as in Fig. 2a), and the discontinuous conduction mode (inductor current becomes zero for a portion of switching period, as in Fig. 2b).

This work was, supported in part by Subcontract No. A72042-RHBE form TRW Systems Group under NASA Prime Contract NAS3-19690, by Subcontract No. D04803-CFCM from TRW Systens Group under NASA Prime Contract NAS3-20102, by NASA through the Jet Propulsion Laboratory of the California Institute of Technology, and by the Naval Ocean Systems Center through MIPR No. N0095377MP09018. a) buck power stage:

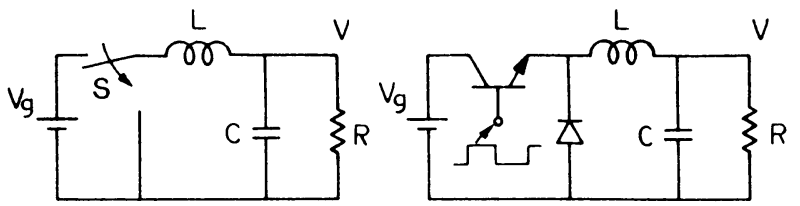

boost power stage:

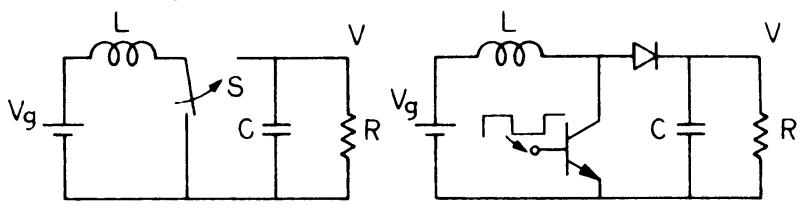

buck-boost power stage:

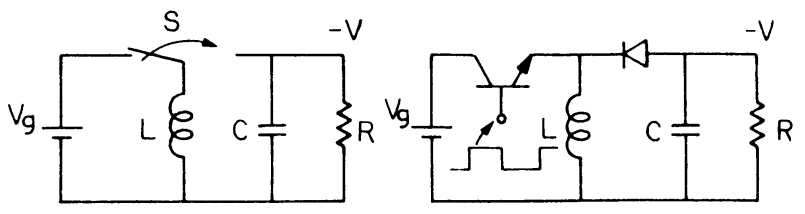

Fig. 1. Three common switching dc-to-dc converters: a) topological configuration independent of switch realization; b) bipolar transistor implementation of the switch $S$.

Consider, for example, the buck-boost converter of Fig. 1 . If the energy stored in the inductor during the first interval DT $\equiv D_{1} T$ is completely released to the output load before the switching cycle $T$ has ended, the inductor current becomes zero for the last portion $\mathrm{D}_{3} \mathrm{~T}$, as seen in Fig. $2 b$.

a)

6)
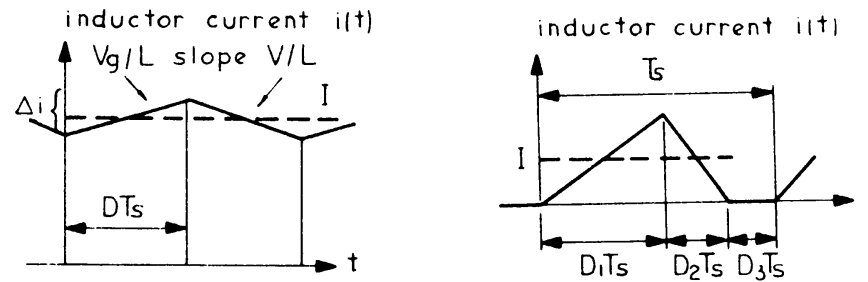

Fig. 2. Inductor current waveforms and definition of two conduction modes: a) continuous conduction mode; b) discontinuous conduction mode. 
Thus the transition from continuous to discontinuous conduction mode is obtained by either increase of load $R$ (hence by lowering of the average dc current $I$ ) or by decrease of inductance $L$ or switching frequency $f_{s}$. In any case, however, the operation in the discontinuous conduction mode results in three different switched networks, as illustrated in Fig. 3 for the buck-boost converter (as opposed to two switched networks for continuous conduction operation). An analogous situation exists for the other two converters of Fig. 1 as well as for a number of other switching converters.

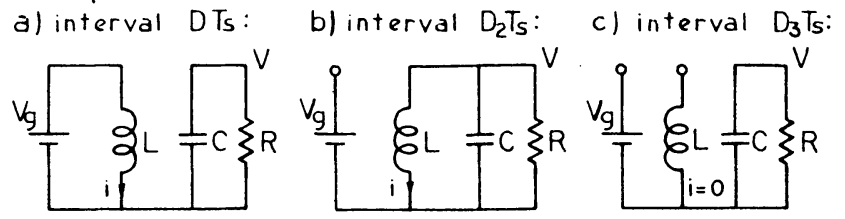

Fig. 3. Three switched networks for the buck-boost converter operating in the discontinuous conduction mode: a) transistor on, diode off; b) transistor 066 , diode on; c) transistor o6f, diode of6.

In Section 2 an extensive overview of the complete structure of modelling of switching converters and regulators in the discontinuous conduction mode by use of the new method is provided. In particular, the steps leading to the equivalent circuit models that describe both steady-state (dc) and dynamic (ac small signal) behaviour are briefly explained. The subsequent sections then give a detalled and thorough account of the new method outlined in Section 2.

First, in Section 3, the procedure for modelling in discontinuous conduction mode is viewed as a special case of that for continuous conduction mode $[1,2,3]$ (provided the state-space averaging step of [2] is properly generalized to include three or more structural changes within each switching period as shown in Appendix) and additional constraints imposed to model special Inductor current behaviour. Though the results obtained are in terms of linear equations, the useful circuit realizations may be obtained as in Section 4. The straightforward perturbation and linearization steps in Section 5 lead to dc and ac circuit models. They result for three common converters of Fig. 1 in the fixed topology, canonical circuit model and are easily tabulated. Because of the need for complete presentation of the theoretical background of the new method, and lack of space, only cursory experimental verification is included at the end of Section 6. Finally, in Section 7 the completeness of the obtained converter circuit models is reemphasized by their direct incorporation in switching regulator models.

Since the method presented here is essentially a consistent extension of the technique for continuous conduction mode [2], the exposition will closely follow the format given in [2], such that the common steps to both methods become immediately transparent, and those that are different clearly distinguished.

\section{REVIEW OF THE NEW STATE-SPACE MODELLING TECHNIQUE} IN THE DISCONTINUOUS CONDUCTION MODE

\subsection{Brief review of existing modelling techniques}

Owing to the relatively more complicated nature of the converter operation in the discontinuous conduction mode, dynamic (ac small signal) models have been lacking (even though valid models for continuous conduction mode have already been obtained) until recently several approaches $([4]-[10])$ have been proposed. However, while all these techniques ([4]-[10]) provide through various linearization procedures the proper linearized transfer functions (duty ratio modulation $\hat{d}$ to output voltage $\hat{v}$ and line voltage $\hat{v}$ to output voltage $\hat{v}$ transfer functions), they are incapable of representing the input properties of the converter, and hence fall to arrive at the complete linearized converter model. This is an entirely analogous situation to that for continuous conduction mode $[2,3]$, where these methods could not model the input properties (open- and closed-loop input impedance, for example) of the converters and regulators in continuous conduction mode of operation. In addition, they stay throughout modelling in the domain of equation manipulations on $1 y$, and thus the useful insight which can be gained from linear circuit models (as demonstrated in $[1,2,3]$ is lost. Hence the primary objective of the development here becomes to overcome all these difficulties by extending the powerful state-space averaging technique of [2], together with its circuit model realizations, to the discontinuous conduction mode of converter operation and finally to arrive at the complete linear circuit model of various converters (1ike, for example, those of Fig. 1).

2.2 New state-space and circuit averaging methods for switching converters in the discontinuous conduction mode

The state-space and circuit averaging methods presented in [2] are now to be suitably modified to account for the discontinuous conduction mode of operation, and the results are summarized in the Flowchart of Fig. 4. As before for the continuous conduction mode, the starting model for the switching converter (block 1 in the Flowchart of Fig. 4) is either in terms of the statespace description of the switched networks (as in block 1a), or in terms of linear circuit models of the switched networks (as in block 1b).

The difference, however, from the previous description is not only that now there are three different structural configurations within each switching period, but also in the fact that instantaneous inductor current is restricted in its behavior: it starts at zero at the beginning of a switching period and falls to zero current again even before the switching period has expired (see the instantaneous inductor current waveform in block 1 of Fig. 4).

It is actually this second difference which clearly distinguishes the discontinuous conduction mode of operation, while the first difference, that of having three different structural configurations, appears in a way to be merely incidental. That is, in Appendix A it is shown that the state-space averaging step of [2] can be directly extended to include "three-state" converters (converters with three structural changes within each switching period), provided such converters are operated in the continuous conduction mode, and any restrictions on state-space variables (inductor currents and capacitor voltages) are avoided: Therefore, our objective in modelling converters operating in the discontinuous conduction mode (and exhibiting "three-state" configuration behavior) becomes that of supplementing this generalized state-space averaging step for "threestate" converters by additional constraints which reflect the special behavior of one of the state variables, the inductor current. Hence the switching-mode converter operating in the discontinuous conduction mode (and having three structural changes) may be viewed as a special case of the ordinary "three-state" converters which are free from any restrictions on state vartables. Thus the primary goal is properly to determine these additional constraints and to find how they propagate through various paths of the modelling (such as paths $a$ and $b$ on the Flowchart of Fig. 4). 
From the Flowchart of Fig. 4 it is immediately clear that path a follows a development strictly in terms of state-space equations, the state-space averaged mode1ling technique, while the other path $b$ proceeds in terms of circuit models, circuit averaged modelling. Moreover, as before for the continuous conduction mode, along path $a$ the general equations (through general matrices $A_{1}, A_{2}, A_{3}$ and vectors $b_{1}, b_{2}$, and $b_{3}$ ) are retained to emphasize the fact that the outlined procedure is applicable to any "three-state" converter operating in the discontinuous conduction mode, while along path $b$ a particular example of the boost con-

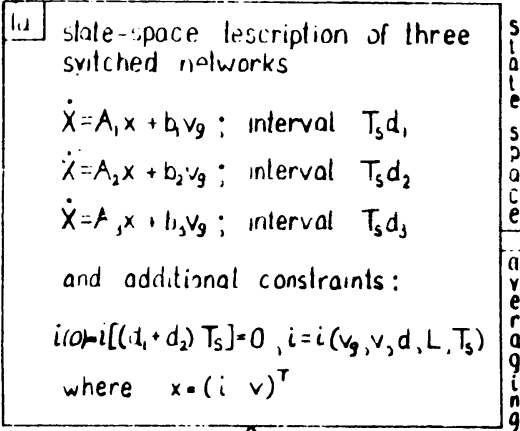

stale-space $\{$ modeling

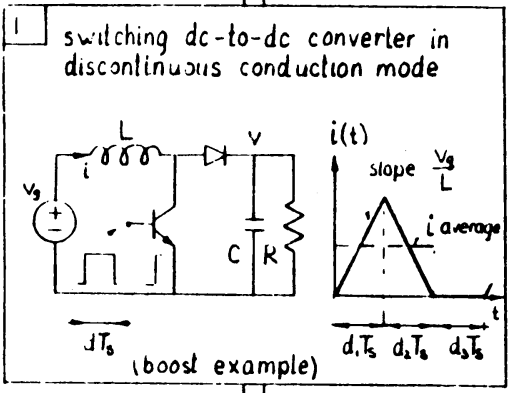
circuit $\sqrt{\text { modeling }}$

It three swilched circuit models: interval $T_{3} d_{1}$ :

$\left.\sum^{2} \gamma_{i}\right] \mathbb{C} S R$ interval $T_{s} d$,
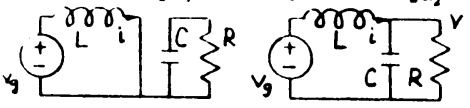
interval $T_{s} d_{3}$

$\left.v_{i} \sum_{C T}^{T}\right\}_{R}$

and addilional constraints:

$i(0)=i\left[\left(d_{1}+d_{1}\right) T_{s}\right]=0, i=\frac{v_{2}}{2 L} d T_{s}$ (boost example)
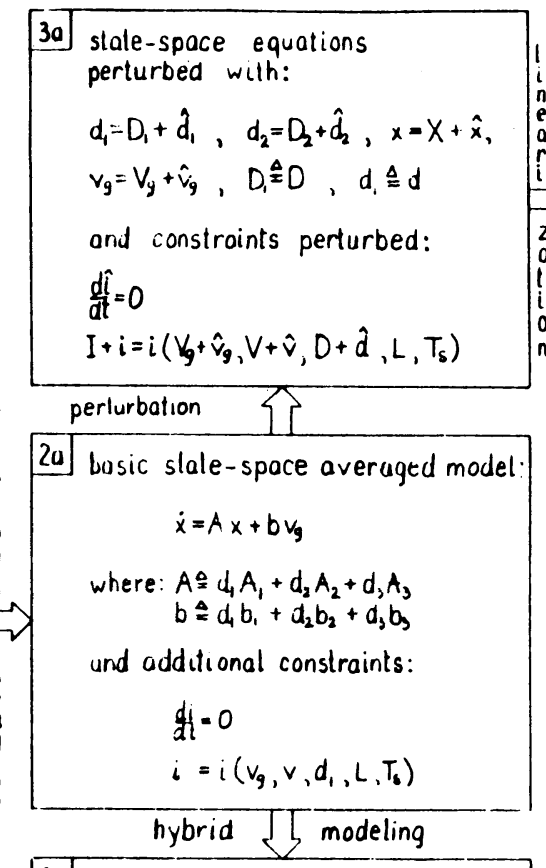

$2 a$ busic slale-space averaged model

$$
\dot{x}=A x+b v_{g}
$$

where: $A \circ d_{1} A_{1}+d_{2} A_{2}+d_{3} A_{3}$ $b \triangleq d_{1} b_{1}+a_{2} b_{2}+a_{3} b_{3}$

und additional constraints:

$$
\begin{aligned}
& d i=0 \\
& i=i\left(v_{g}, v, d_{1}, L, T_{8}\right)
\end{aligned}
$$

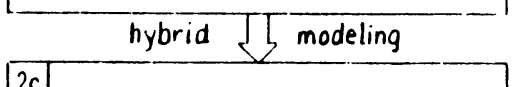

stole-space equations

in a circuit recognizable

form

(stale-space realization) circuit $\int$ recognilion

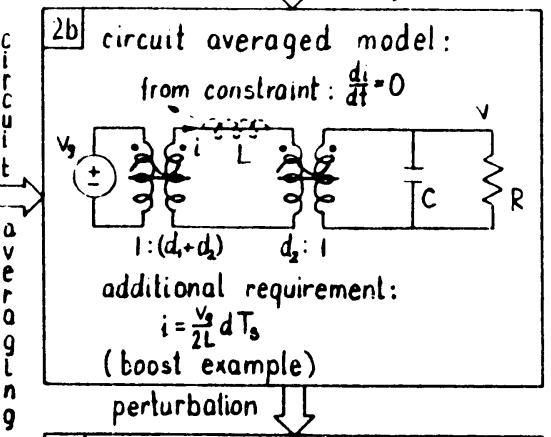

36 circuit model perlurbed:

with: $d-D+d, d_{2}=D_{2}+a_{2}, i=I+\hat{i}$

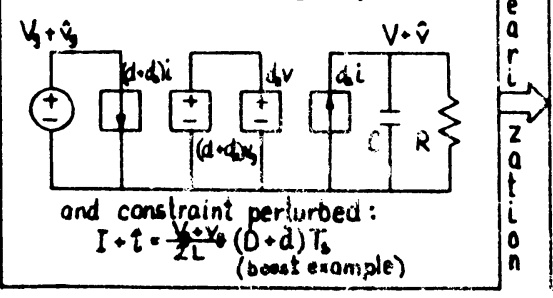

4a final stale-space averaged model: steady state (dc) model:

$X=-A^{-1} b V_{y}$ with $I=i\left(V_{g}, V, D, L, T_{s}\right)$ dynamic ( $a c$ ) small signal model: $\dot{\hat{x}}=A \hat{x}+b \hat{v}_{9}+d_{1}\left[\left(A_{1}-A_{3}\right) X+\left(b_{1}-b_{3}\right) V_{g}\right]+$ $+d_{2}\left[\left(A_{2}-A_{3}\right) X+\left(b_{2}-b_{3}\right) V_{9}\right]$ with: $\frac{d \hat{i}}{d \hat{t}}=0$ $\hat{\imath}=\frac{\partial i}{\partial v_{j}} \hat{v}_{y}+\frac{\partial i}{\partial v} \hat{v}+\frac{\partial i}{\partial d} \hat{d}$

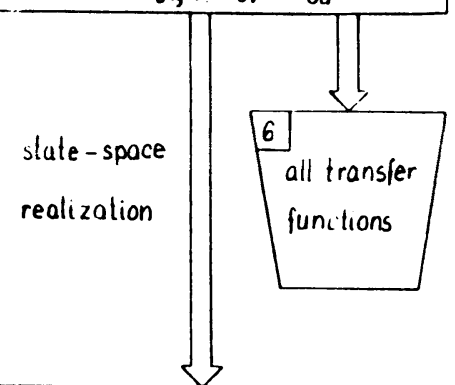

5 canonical circuit model steady slate $(d c)$ model:

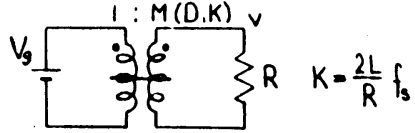

dynamic (ac small-signal) model:
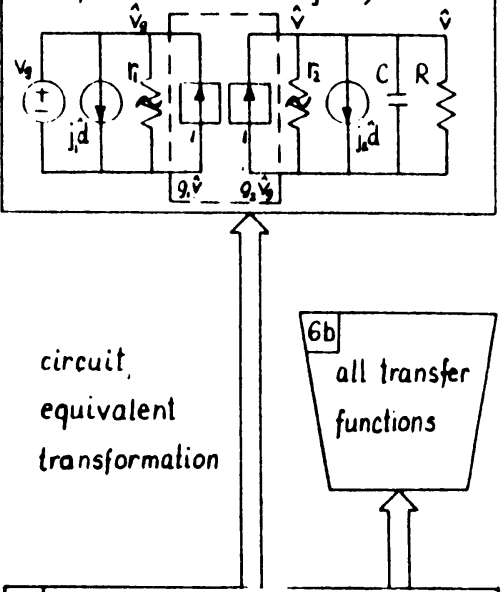

16 final averaged circuit models steady-state $(d c)$ model

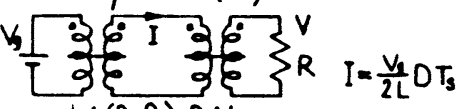

$1:\left(Q_{+}+Q_{)}\right) D_{1}: 1$ dynamic (ac small-signal) model

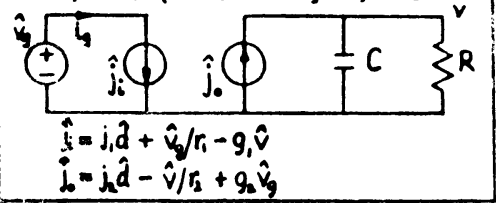

Fig. 4. Flowchart of averaging approaches in modelling switahing dc-to-ic converters in the discontinuors conduction mode. Path a: general staie-space midelivig; Path b: circuit transformation method. 
verter is followed, owing to the requirement for the specific converter topology along that path. Specifically, for the boost power stage, $A_{1}=A_{3} \neq A_{2}$ are $2 \times 2$ matrices, and $b_{1}=b_{2} \neq 0, b_{3}=0$ are vectors. This example will later be pursued in detall along both paths.

We now follow path a more closely. The cruclal step is made in going from block la to $2 a$ in that the original description through three state-space equations (block 1a) is substituted by a single state-space averaged mode1 (block $2 a$ ). This is justified as follows. The fundamental performance requirement of switching converters (negligible switching ripple) results in natural frequencies $\omega_{\alpha}$ and $f$ much lower than the switching frequency $f{ }^{\alpha}$ This, ${ }^{c}$ in turn, leads as shown in Appendix A to the generalized state-space averaging step. So far this would be the same averaging step as applied to any ordinary "three-state" switching converter. However, as indicated before, the inductor current 1 does not behave as a true statespace variable in the discontinuous conduction mode since it does not have free boundary conditions (but fixed at zero) which is shown to lead to the following constraint:

$$
\frac{d 1}{d t} \equiv 0
$$

This immediately reduces by one the order of the basic state-space averaged model (block $2 a$ ), since one of the dynamic equations (that for inductor current) reduces to a static equation. In addition to this, an expression describing the average inductor current $i$ can be found directly from the converter itself (block 1 ) and becomes the second constraint, termed perturbation equation $I$, which is

$$
i=i\left(v_{g}, v, d, L, T_{s}\right)
$$

Thus, the two additional constraints (1) and (2), together with the generalized state-space averaging step, completely determine the converter model in the discontinuous conduction mode. It remains only to apply the standard perturbation techniques (block 3a) and (on the basis of the small-signal assumption) the linearization techniques to both state-space averaged equations and the perturbation equation of block $2 a$ in order to arrive at the final state-space averaged model (block 4a). This model gives separately both dc and ac small-signal descriptions through general matrices $A_{1}, A_{2}, A_{3}$ and vectors $b_{1}, b_{2}, b_{3}$ of the starting switched models (block $1 \frac{1}{a}$ ) and constraints corresponding to those of (1) and (2).

Naturally, we can now proceed from the basic statespace averaged model (block $2 a$ ) via hybrid modelling and circuit recognition (block $2 c$ ) to arrive at the very useful circuit realization (block 2b). Note, however, that now the constraint (1) effectively leads to shorting the inductance $\mathrm{L}$ in the circuit model since $v_{\mathrm{L}}=\mathrm{L} \mathrm{d} 1 / \mathrm{dt}=0$ This, for the particular boost circuit example, reduces the circuit to first order. The other constraint (2) is also easily specified (see additional constraint in block 2b) with the help of the inductor current waveform (block 1). The same c1rcuit model (block 2b) could, however, be obtained directly from the switched circuit models (block $1 \mathrm{~b}$ ), by following the circuit averaging path, provided the circuit averaging step for "threestate" converters is supplemented by the aforementioned equivalents of the constraints (1) and (2). Again, the remaining circult perturbation (block $3 b$ ) and circuit linearization steps are straightforward and result in the final circuit averaged models (block $4 \mathrm{~b}$ ) separately for $d c$ and ac sma11-signal. As seen from block 4b, the dc part of the perturbation equation, current $I$, togethe with the dc circuit model, completely determines the dc conditions, while its ac part $\hat{i}$ contributes to the final ac circuit averaged model.
Finally, both models (block $4 \mathrm{a}$ or $4 \mathrm{~b}$ ) can be used to determine the transfer functions of interest: line voltage variation $\hat{v}_{\text {and }}$ auty ratio modulation $\hat{\mathrm{d}}$ to output voltage $\hat{v}$ (bfocks $6 a$ and $6 b$ respectively).

\subsection{New canonical circuit model for discontinuous conduction mode}

As for the continuous conduction mode, the culmination of the modelling is again a canonical circuit model (block 5 of Fig. 4), whose fixed topology (though different from the one for continuous conduction mode) has all the features necessary to present a complete circuit model. However, th1s fixed topology of the model for discontinuous conduction mode came merely as a by-product, since for the three converters of Fig. 1 (buck, boost, and buck-boost) the ac small-signal models all resulted in the fixed topological structure of the model in block 4b of Fig. 4 without any need for equivalent circuit or other transformations. It does not appear that this canonical c1rcuit topology could be directly extended to some arbitrary converter. Even though this canonical circuit model is not so general as that for two-state converters [2], a useful comparison between the two canonical circuit topologies can be made (at least for the common converters of Fig. 1 in both operating modes).

While in the continuous conduction mode the effect of duty ratio modulation $\hat{\mathrm{d}}$ was represented by voltage and current duty ratio dependent generators at the input port (hence properly representing negative closedloop input impedance at low frequencies as shown in [2], here in discontinuous conduction mode there are two duty ratio dependent current generators, one in the input circuit (again, properly to model converter input properties as shown later in Section 7 ), and the other in the output circuit to generate the duty ratio $a$ output transfer function.

The salient feature of the canonical circuit model in block 5 of the Flowchart in Fig. 4 is that both transfer functions are obtained using only the output port of the complete canonical circuit model, unlike the situation for continuous conduction mode where the complete circult model was necessary to determine them. This is also why other methods which properly represent the transfer function in discontinuous conduction mode ([4]-[10]) have completely omitted modelling of the converter input properties.

\subsection{Extension to complete regulator treatment}

It w111 be shown in Section 7 how the linear model of the modulator stage can be obtained. It remains simple to incorporate the canonical circuit model (block 5 in the Flowchart of Fig. 4) to arrive at the linear circuit model of a closed-loop switching regulator operating in the discontinuous conduction mode.

A word of caution, however, is appropriate here. Namely, since the very nature of operation in the discontinuous conduction mode 18 that the order of the system is reduced at least by one, this would definitely change the dynamics and possible compensation networks necessary for stable operation of the closed-loop regulator. Furthermore, if both conduction modes are expected to take place for the particular application, the compensation network should be designed to ensure stability of the closed-loop and acceptable transient performance for either of the two modes. Hence canonical circuit models for both continuous and discontinuous conduction mode become an invaluable tool in the proper design of switching regulators. In addition, comparison of the advantages and/or disadvantages between the two modes of operation become feasible, and possible trade-offs between regulator performance and choice of parameters and operating conditions is cleariy displayed. 
In summary, the new method is generally applicable to any "three-state" converter operating in the discontinuous conduction mode (block $4 a$ ), even though for an arbitrary converter the final circuit model (block 4b) may have different (more complicated) topology than the canonical circult model for the three common converters (block 5). We also emphasize the fact that the methods for finding dc and ac small-signal models are consistent with each other. Namely, for both models we need only the standard state-space or clrcuit averaging step (depending on whether path $a$ or $b$ is chosen) applicable to any converter with three switched network configurations. Then to distinguish that the converter is operating in the discontinuous conduction mode, additional restrictions (1) and (2) are 1mposed. Now, the dc part of perturbation equation (2) together with the dc state-space or circuit averaged model completely determines the final dc model, while the ac part $\hat{I}$ of (2) helps in complete definition of the final ac small-signal state-space or circult averaged model.

It may seem that the method outlined holds only for "three-state" converters in discontinuous conduction mode. This is not so, since it can easily be generalized to include more complicated schemes of discontinuous conduction mode of operation. As an 1llustration of this generality, consider the new class of switching converters of Appendix A, the cascade connection of ordinary buck and boost converters, which could also be classified as two-Inductor converters ( as opposed, for example, to the converters of Fig. 1 which are one-inductor converters). Suppose also that the two switches are driven synchronously with the same switch duty ratio $D$, thus resulting in a two-state converter for continuous conduction operation. If, however, one of the two inductor currents becomes discontinuous, a three-state converter operating in the discontinuous conduction mode is obtained. But now the matrices $A_{1}, A_{2}, A_{3}$ and $A$ would be of 4 th order (as opposed to $2 \mathrm{nd}$ order for the converters of Fig. 1) and the final state-space or circuit averaged model would be of the 3rd order (reduction of order by one due to discontinuity of one of the two inductor currents). Moreover, there is also the possibility that both inductor currents could become discontinuous under certain operating conditions in which case four-state converters are generated. Therefore, the generalized state-space averaging step (Appendix A) applicable to four-state converters is supplemented with additional constraints: for each discontinuous current there will be two constraints imposed analogous to (1) and (2). The immediate consequence of these constraints is that the fourth-order original converter model becomes only a second-order final state-space or circult averaged model (with two inductances effectively disappearing from the final circuit averaged mode1).

Despite this demonstration of the generality of the method, we will restrict ourselves in the remaining Sections to the "three-state" converters in the discontinuous conduction mode since all the essential features of the method are present there.

\section{STATE-SPACE AVERAGING IN DISCONTINUOUS CONDUCTION MODE}

Various paths on the Flowchart of Fig. 4 will now be followed in detail, first with general derivation and then fllustrated by examples.

\subsection{State-space averaging}

In this section, the final state-space averaged model (block 4a of Fig. 4) is derived, first in general for any three-state switching converter in discontinuous conduction mode, and then demonstrated on the idealized boost circuit example (parasitic effects not included). Steady state (dc) conditions are obtained

for this particular example and discussed in depth, including determination of the boundary between the two modes of converter operation.. From the dynamic (ac smal1signal) model, the two transfer functions of interest $(\hat{v}(s) / \hat{v}(s)$ and $\hat{v}(s) / \partial(s))$ are also determined to enable comparison with the corresponding transfer functions derived from the final circuit averaged model for the boost converter presented in Section 3.3.

\section{Basic state-space averaged model}

We first define the time-domain description of an arbitrary three-state switching converter operating in the discontinuous conduction mode with the help of Fig. 5, which displays the switch drive (Fig. 5a) and instantaneous Inductor current (F1g. 5b) which becomes discontinuous. The definition of the three intervals $T_{8} d_{1}, T_{8} d_{2}$, and $\mathrm{T}_{\mathrm{S}} \mathrm{d}_{3}$ (or corresponding steady-state quantities ${ }^{8} \mathrm{~T}_{\mathrm{B}} \mathrm{D}_{1}$, $T_{s}^{s} D_{2}^{3}$, and $T_{s} D_{3}$ ) is also clearly visible on Fig. $S^{1}$

a)

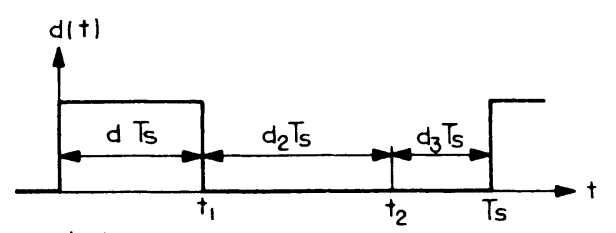

b)

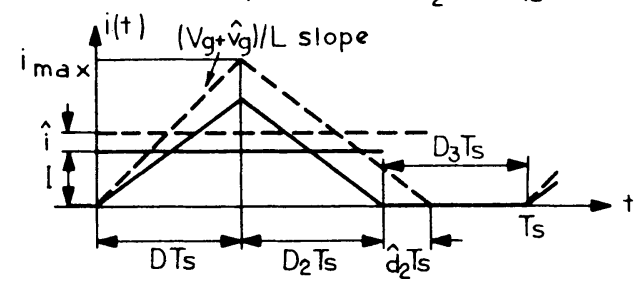

Fig. 5. Definition of the time intervals and perturbation quantities: a) transistor switch drive; b) instantaneous inductor current.

As seen from Fig. 5, the "off" interval $\left[t_{1}, T_{8}\right]$ Is now subdivided into two intervals $\mathrm{T}_{\mathrm{S}} \mathrm{d}_{2}$ and $\mathrm{T}_{\mathrm{d}_{3}}{ }^{8}$ (or $T D_{2}$ and $\left.T_{S_{3}}\right)$. While the first "on" Interval $T$ is dickated by the switch drive and is a known quantity (at least in open-loop converter usage), the second interval $\mathrm{T}_{s} \mathrm{~d}_{2}$ (or $\mathrm{T}_{s} \mathrm{D}_{2}$ ), which will be termed the "decay" interval, is as yet unknown and depends in general on both the length of the first interval and some circuit parameters, and describes how deep in the discontinuous conduction mode the converter is operating. Nevertheless we assume that the decay interval $T D_{2}$ exists (hence the discontinuous conduction mode) and Ieave it to the mode1ling procedure itself to reveal how it is actually determined.

For each of the three intervals in Fig. 5, there exists in general a different switched network (compare with Fig. 3 for the buck-boost converter example), which can be described by a corresponding state-space equation as follows:

$$
\begin{array}{lll}
\dot{x}=A_{1} x+b_{1} v_{g} & \text { for interval } d_{1} T_{s}, & \left(0 \leq t \leq t_{1}\right) \\
\dot{x}=A_{2} x+b_{2} v_{g} & \text { for Interval } d_{2} T_{s}, & \left(t_{1} \leq t \leq t_{2}\right) \\
\dot{x}=A_{3} x+b_{3} v_{g} & \text { for Interval } d_{3} T_{s}, & \left(t_{2} \leq t \leq T_{s}\right)
\end{array}
$$

While for the continuous conduction mode a similar expression is sufficient to describe the converter, here in discontinuous conduction mode, (3) does not describe the switching converter completely. Namely, the instantaneous inductor current is restricted in its evolution since from Fig. 5

$$
i(0)=i\left[\left(d_{1}+d_{2}\right) T_{s}\right]=0 \text { and } i(t) \equiv 0 \text { for } t \varepsilon\left[t_{2}, T_{8}\right]
$$


Therefore (3) together with (4) completely determine the behavior of the switching converter. However, directly from this description, even the determination of the steady-state (dc) conditions on an exact basis might be a very difficult (if not insurmountable) task, and moreover the tremendous complexity of the result may be unnecessary. In addition, the direct perturbation of (3) and (4) to obtain the dynamic response of the converter would become by an order of magnitude more difficult if not virtually impossible. Our objective then becomes, as 1t was in [2] for the continuous conduction mode, to replace the original converter description through three state-space equations (3) by a single state-space descrip tion which will accuratley represent the evolution of the state-vector at the switching instants. It is also desir able that the additional constraint (4) be appropriately accounted for to modify this averaging equivalent, but in such a way as to interfere the least possible with its orderly procedure.

The first task is accomplished by application of the generalized state-space averaging step for three-state converters (Appendix A) to (3), which results in a single state-space description

$$
\dot{x}=\left(d_{1} A_{1}+d_{2} A_{2}+d_{3} A_{3}\right) x+\left(d_{1} b_{1}+d_{2} b_{2}+d_{3} b_{3}\right) v_{g}
$$

Note, however, that this continuous description is a continuous equivalent to the originally derived approximate discrete system [1]. Hence the definition of a discrete derivative [1] transforms the constraint (4) into

$$
\frac{d 1}{d t}\left(\mathrm{nT}_{8}\right)=\frac{1\left(\mathrm{~T}_{8}\right)-1(0)}{\mathrm{T}_{8}}=0
$$

It follows that the inductor current in the equivalent continuous system (5) ceases to be a true statespace variable, since according to (6) it has lost its dynamic properties. Nevertheless, despite the zero constraints $1\left(\mathrm{nT}_{\mathrm{s}}\right)=0$ and $\mathrm{di} / \mathrm{dt}(\mathrm{nT})=0$ for $\mathrm{n}=0,1, \ldots$, a line voltage perturbation $v_{\text {(as seen }}$ (n Fig. $5 \mathrm{~b}$ ) does cause a perturbation of the ifistantaneous inductor current (shown in dotted lines on Fig. 5b) from 1ts steadystate waveform (heavy line in Fig. 5b), which in turn results in a corresponding perturbation $\hat{v}$ of the output steady-state voltage. Note that there is also perturbation of the average inductor current 1 (defined in Fig. $5 b$ for interval $\left(d_{1}+d_{2}\right) T$ when instantaneous inductor current $1(t)$ is different trom zero) from its steadystate average current $I$. This is in sharp contrast to the situation in the continuous conduction mode where the average inductor current does not change under any smal1signal perturbation, but rather initial and final conditions $i(0)$ and $i\left(T_{s}\right)$ change accordingly to accommodate perturbation. Here, $1(0)$ and $1\left(T_{s}\right)$ are fixed at zero. and the average inductor current 18 the quantity which reflects the effect of introduced perturbation.

Since the objective in modelling the dynamic performance of the converter is faithfully to represent departure from the steady-state, we introduce the average Inductor current as a substitute for the "lost" statevariable (the instantaneous inductor current). But, rather than change the symbol, we assign to the same designation $i$ this new meaning. Then from Fig. $5 b$ we obtain

$$
1=\frac{1}{2}=1\left(v_{g}, v, d, L, T_{s}\right)
$$

and designate it perturbation equation $I$, for reasons which will become apparent later. Naturally, the other constraint (6) for this average inductor current 1 is maintained (as seen also from Fig. 5b) and we finally obtain the basic state-space averaged model for discontinuous conduction mode:

$$
\dot{x}=\left(d_{1} A_{1}+d_{2} A_{2}+d_{3} A_{3}\right) x+\left(d_{1} b_{1}+d_{2} b_{2}+d_{3} b_{3}\right) v_{g}
$$

with additional constraints

$$
\begin{aligned}
\frac{d i}{d t} & =0 \\
1 & =1\left(v_{g}, v, d_{1}, L, T_{s}\right)
\end{aligned}
$$

The two additional constraints (9) and (10) modify the ordinary averaged model (8) to account for the discontinulty of the 1nductor current. This mode1 (block 2a in the Flowchart of Fig. 4) is the starting point for all other derivations (both state-space and circuit-oriented) and represents an averaged model over a single period $T_{8}$.

Note, also from (7) that the caluclation of the average inductor current 1 is actually based on the assumption of the linearity of the inductor current waveform (triangular waveshape in Fig. 5). However, this does not pose any limitations at all, since the linearity of the inductor waveform is again a consequence of the small switching ripple requirement and therefore consistent with the same basic assumption made in the continuous conduction mode.

We now consider first the simplest possible case, determination of the basic dc conditions in the steady state regime. In the steady state all quantities become dc quantities and are denoted by capital letters, that is, $d_{1}=D_{1}=D, d_{2}=D_{2}, d_{3}-D_{3}, v_{0}=v_{p}, x=x$. The average inductor current i becomes the steady state average inductor current I (see Fig. 5b, for example) and the steady-state vector $X=(I \quad V \ldots)$. Since then $\mathrm{dX} / \mathrm{dt} \equiv 0$, the state-space equation (8) reduces to the linear algebraic system

$$
A X+b V_{8}=0
$$

where

$$
\begin{aligned}
& A=D_{1} A_{1}+D_{2} A_{2}+D_{3} A_{3} \\
& b=D_{1} b_{1}+D_{2} b_{2}+D_{3} b_{3}
\end{aligned}
$$

while the first constraint (9) is automatically satisfied and the second constraint becomes

$$
I=I\left(V_{8}, V, D_{1}, L, T_{s}\right)
$$

It is now interesting to compare these results for dc conditions (11), (12) and (13) with those for the continuous conduction mode [2]. For easier correlation of these results, the notation $d_{1}=d$ and $D_{1}=D$ henceforth will be used interchangeably. ${ }^{1}$ The steady state vector $X$ is the solution of the linear system (11) as 1 t was before in [2]. Hence storage elements ( $L$ 's and $C^{\prime} s$ ) are proportionality constants in the linear system (11) and it appears as though solution $X$ of (11) is independent of them and dependent on dc duty ratios and resistances in the original mode1. However, since $D_{1}+D_{2}+D_{3} \equiv 1$ or $D_{3}=1-\left(D+D_{2}\right)$ from (11) and (12) it follows that the steady state vector $X$ is now dependent on two duty ratios $D$ (given) and $D_{2}$ (as yet undetermined) as opposed to only $D$ in [2]. The additional constraint (13) which expresses the average steady state inductor current I in terms of circuit parameter values can now be used together with (11) to solve for the unknown duty ratio $D_{2}$, and hence to determine the length of the second interval $\mathrm{D}_{2} \mathrm{~T}$. In general, then, $D_{2}$ is dependent on circuit parameters (such as $\mathrm{L}$ and $\mathrm{T}$, for example) and hence dc conditions are also substantialiy dependent on switching frequency $f_{8}$ and inductance $L$. This is in sharp contrast to the continuous conduction mode [2], where dc conditions are dependent on duty ratio $D$ and resistances on1y. 
In summary, expressions (11) and (13) completely determine the dc conditions in the discontinuous conduction mode, and at the same time help to determine the length of the second interval $\mathrm{D}_{2} \mathrm{~T}_{s}$, which was unknown at the beginning of this analysis.

We now undertake to obtain the dynamic model by perturbation of the basic model $(8-10)$.

\section{Perturbation}

Suppose that the switch drive duty ratio $d$ changes from cycle to cycle, in addition to the line voltage variation. Hence, the general perturbation equations

$$
\begin{aligned}
& d=D+\hat{d} \\
& \mathrm{~d}_{2}=\mathrm{D}_{2}+\hat{\mathrm{d}}_{2}, \\
& d_{3}=D_{3}+\hat{d}_{3}, \\
& \hat{v_{g}}=v_{g}+v_{g}, \\
& x=x+\hat{x} \text {, } \\
& \text { and } i=I+\hat{i}
\end{aligned}
$$

introduced into the basic-state space averaged model given by $(8),(9)$, and (10) result in

$$
\begin{aligned}
\dot{\hat{x}} & =\left[(D+\hat{d}) A_{1}+\left(D_{2}+\hat{d}_{2}\right) A_{2}+\left(D_{3}-\hat{d}-\hat{d}_{2}\right) A_{3}\right](x+\hat{x})+ \\
& +\left[(D+\hat{d}) b_{1}+\left(D_{2}+\hat{d}\right) b_{2}+\left(D_{3}-\hat{d}-\hat{d}_{2}\right) b_{3}\right]\left(v_{g}+\hat{v}_{g}\right)
\end{aligned}
$$

with additional constraints

$$
\begin{aligned}
\frac{d \hat{i}}{d t} & =0 \\
I+\hat{I} & =I\left(V_{g}+\hat{t}_{g}, V+v, D+\hat{d}, L, T_{s}\right)
\end{aligned}
$$

From $d+d_{2}+d_{3} \equiv 1$, when perturbed by (14), we got $\mathrm{D}+\hat{\mathrm{d}}+\mathrm{D}_{2}{ }^{2}+\hat{\mathrm{d}}_{2}{ }^{3}+\mathrm{D}_{3}+\hat{\mathrm{d}}_{3} \equiv 1$ or, since also $\mathrm{D}+\mathrm{D}_{2}+$ $\mathrm{D}_{3} \equiv 1$, we finally arrive at

$$
\hat{\mathrm{d}}_{3}=-\left(\hat{\mathrm{d}}+\hat{\mathrm{d}}_{2}\right)
$$

which was then used in (15).

The perturbed model given by (15), (16), and (17) is nonlinear owing to the presence of at least secondorder terms.

Linearization and final state-space averaged model for discontinuous conduction mode

We now make the small-signal approximation, namely that the departures from the steady-state values are small compared to the steady-state values themselves:

$$
\frac{\hat{\mathrm{v}}_{\mathrm{g}}}{\mathrm{v}_{\mathrm{g}}} \ll 1, \quad \frac{\hat{\mathrm{d}}}{\mathrm{D}} \ll 1, \quad \frac{\hat{\mathrm{d}}_{2}}{\mathrm{D}_{2}} \ll 1, \quad \frac{\hat{\mathrm{x}}}{\overline{\mathrm{x}}} \ll 1
$$

Using approximations (19) we neglect all second (or higher) order terms, and obtain once again a linear system but including duty-ratio modulation d. After separating the steady-state (dc) and dynamic (ac) parts of both state-space equations (15) and constraints (16) and (17) we arrive at the following results for the final state-space averaged model.

Steady state $(d c)$ model:

$$
\mathrm{x}=-\mathrm{A}^{-1} \mathrm{bv}_{\mathrm{g}}
$$

Subject to constraint

$$
I=I\left(V_{g}, V, D, L, T_{s}\right)
$$

Dynamic (ac small-signal) model:

$$
\dot{\hat{x}}=A \hat{x}+b \hat{v}_{g}+\hat{d}\left[\left(A_{1}-A_{3}\right) x+\left(b_{1}-b_{3}\right) v_{g}\right]+\hat{d}_{2}\left[\left(A_{2}-A_{3}\right) x+\left(b_{2}-b_{3}\right) v_{g}\right]
$$

subject to constraints

$$
\begin{aligned}
& \frac{d \hat{i}}{d t}=0 \\
& \hat{i}=\frac{\partial i}{\partial v_{g}} \hat{v}_{g}+\frac{\partial i}{\partial v} \hat{v}+\frac{\partial i}{\partial d} \hat{d}
\end{aligned}
$$

where $A$ and $b$ are as given before by (12).

From (24) it also becomes obvious why (7) was originally called "perturbatiop equation I." In addition, since $\hat{x}=[d \hat{i} / d t \hat{d v} / d t \ldots]^{T}$ the introduction of constraint (23) into (22) reduces the first dynamic equation to a static one, from which the unknown modulation $\hat{d}_{2}$ can be determined in terms of $\hat{v}_{g}$ and $\hat{i}$ modulations and circuit parameters.

The dynamic state-space equation which, because of (23), became a static one, can now be designated "perturbation equation II," since it helps to determine the other unknown perturbation quantity $d_{2}$. Together with (24) this uniquely defines the line transfer function $\hat{\mathrm{y}}(8) / \mathrm{v}(s)$ and duty ratio modulation transfer function $\hat{v}(s) / \hat{d}(s)$. However, owing to the presence of constraints (23) and (24) no closed-form expression is avallable for the transfer functions, unlike the case for the continuous conduction mode.

We conclude this section with illustration of these general results on the boost converter. Both dc and ac small-signal models are then analyzed in detail and some unique insights into the operation of the boost converter in the discontinuous conduction mode are obtained. Dc conditions and the determination of the boundary of the two modes of operation are particularly thoroughly analyzed.

Example: ideal boost power stage in discontinuous conduction mode

For the 1deal boost power stage of Fig. 1 the three switched networks in the discontinuous conduction mode of operation are shown in Fig, 6 .

a) interval dTs: b) interval $d_{2} T_{s}$ : c) interval $d_{3} T_{s}$ :

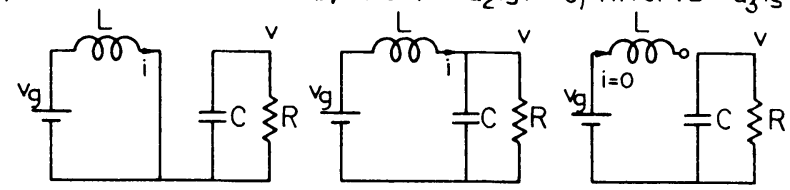

Fig. 6. Three switched networks of the ideal boost converter of Fig. 1 operating in the discontinuous conduction mode.

For the choice of state-space vector $x=\left(\begin{array}{ll}1 & v\end{array}\right)^{T}$, the state-space equations of the three linear switched networks in Fig. 6 become:

$$
\begin{array}{ll}
\dot{x}=A_{1} x+b_{1} v_{g} & \text { for interval } d_{s} \\
\dot{x}=A_{2} x+b_{2} v_{g} & \text { for interval } d_{2} T_{2} \\
\dot{x}=A_{3} x+b_{3} v_{g} & \text { for interval } d_{3} T_{s}
\end{array}
$$

where 
$A_{1}=\left[\begin{array}{cc}0 & 0 \\ 0 & -\frac{1}{R C}\end{array}\right] \quad A_{2}=\left[\begin{array}{cc}0 & -\frac{1}{L} \\ \frac{1}{C}-\frac{1}{R C}\end{array}\right] \quad A_{3}=\left[\begin{array}{cc}0 & 0 \\ 0 & -\frac{1}{R C}\end{array}\right]$

$b_{1}=\left[\begin{array}{ll}\frac{1}{L} & 0\end{array}\right]^{\mathrm{T}} \quad \mathrm{b}_{2}=\left[\begin{array}{ll}\frac{1}{\mathrm{~L}} & 0\end{array}\right]^{\mathrm{T}} \quad \mathrm{b}_{3}=\left[\begin{array}{ll}0 & 0\end{array}\right]^{\mathrm{T}}$

In addition to this, perturbation equation $I$ (7) is needed. However, it can easily be found from Fig. 6a as

$$
i=\frac{i_{\max }}{2}=\frac{v_{g}}{2 L} d T_{s}=i\left(v_{g}, d, L, T_{s}\right)
$$

The same result could have been concluded also from Fig. 5b, tained which actually represents instantaneous inductor current for the boost converter (or buck-boost converter since both have the same slope during interval $\mathrm{dT}_{8}$ ).

Equations (26) and (27) contain now all that is needed to determine both dc and ac small-signal models by application of the general result, equations (20) through (24). We first analyze in greater depth the steady-state (dc) model.

\section{Steady state (dc) model analysis}

By use of (26) in (20) the following linear algebraic system results
$\underline{A}$

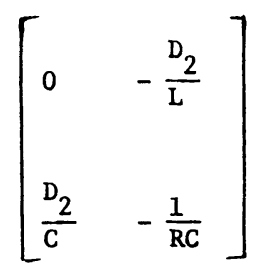

$\underline{\mathrm{x}}$

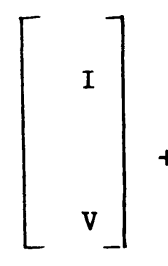

$\underline{\mathrm{b}}$

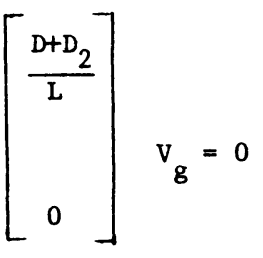

in which the quantities $A, X$ and $b$ are clearly identified and obtained by use of their definition (12). The general remark made previously about the solution of this linear algebraic system (28) becomes clearly visible. Storage elements ( $L$ 's and $C^{\prime} s$ ) are indeed proportionality constants, and the solution of $(28)$ is

$$
\begin{aligned}
& \frac{\mathrm{V}}{\mathrm{V}_{\mathrm{g}}}=1+\frac{\mathrm{D}}{\mathrm{D}_{2}} \\
& \mathrm{I}=\frac{\mathrm{V}}{\mathrm{D}_{2} \mathrm{R}}
\end{aligned}
$$

Hence, the dc conditions depend only on duty ratios $D$ and $D_{2}$ and resistance $R$. From (29) we conclude also that the boost converter has even in the discontinuous, conduction mode the boosting property (dc gain $V / V_{g} \geq 1$ ), since $D, D_{2}$ are by definition positive quantities. However, the ac conditions are not quite determined since $D_{2}$ is as yet unknown. But, by use of the additional constraint (21), as further specified in (27) as

$$
I=\frac{V_{g} T_{s}}{2 L}
$$

together with (29) and (30), dc conditions (and also $\mathrm{D}_{2}$ ) are completely determined. For example, substitution of (31) into (30) results in

$$
\mathrm{D}_{2}=\frac{\mathrm{V}}{\mathrm{RI}}=\frac{\mathrm{V}}{\mathrm{R}} \frac{2 \mathrm{~L}}{\mathrm{DT} \mathrm{V}_{\mathrm{g}}}=\frac{\mathrm{V}}{\mathrm{V}_{\mathrm{g}}} \frac{\mathrm{K}}{\mathrm{D}}=\frac{\mathrm{MK}}{\mathrm{D}}
$$

where the important dimensionless quantity $K$ is defined as

$$
\mathrm{K} \triangleq \frac{2 \mathrm{~L}}{\mathrm{RT}} \mathrm{s} \triangleq \frac{2 \mathrm{~L}}{\mathrm{R}} \mathrm{f}_{\mathrm{s}}
$$

This dimensionless parameter $K$ plays a key role in the discontinuous conduction mode since it combines uniquely all the parameters responsible for such behavior. Another quantity which will frequently appear is the dc voltage gain $\mathrm{V} / \mathrm{V}$, so we define also another dimensionless parameter $\mathrm{M}^{\mathrm{g}}$ as

$$
M \triangleq \frac{V}{V_{g}}
$$

Finally, by use of (32) and (34) in yet unused dc relation (29), the quadratic equation for dc gain $M$ is ob-

$$
M^{2}-M-D^{2} / K=0
$$

Since from (29) the dc gain $M$ is positive, only the positive solution of (35) is meaningful and we obtain

$$
M=\frac{1+\sqrt{1+4 D^{2} / K}}{2}
$$

Finally, the substitution of (36) in (32) determines the previously unknown duty ratio $\mathrm{D}_{2}$ as

$$
D_{2}=\frac{K}{D} \frac{1+\sqrt{1+4 D^{2} / K}}{2}
$$

Hence, we have succeeded in expressing, through (36) and (37), two important quantities, the dc gain $M$ and duty ratio $D_{2}$, in terms of the driving condition (duty ratio $D$ of the transistor switch), and the single dimensionless quantity $K$ which solely reflects the effect of circuit parameter values ( $L$ and $R$ ) and the other operating condition, the switching frequency $f_{s}$, upon the dc conditions in the discontinuous conduction mode. If desired, the remaining dc quantity, the steady-state average inductor current $I$, may be found in terms of $D$ and $K$ by use of (37) in (30).

All these expressions (36), (37), and (30) are very useful in predicting the dc conditions when the switching converter is used alone, that is in an open-loop fashion, since the duty ratio $D$ is given (independently generated) and the constant $K$ may be calculated from element values with use of (33). However, if the converter is used in a closed-loop switching regulator the output dc voltage $V$ is predetermined by the choice of the reference voltage and kept constant regardless of any variation of input dc voltage $v_{\rho}$, by appropriate self-adjustment of the dc duty rat ${ }^{\circ} \mathrm{D}$ (Internally generated) in a negative feedback manner. Hence in closed-loop operation, $\mathrm{D}$ and $\mathrm{D}_{2}$ become dependent on the external dc gain $M$ and the dimensionless parameter $K$. These dependences can easily be found from (36) and (37) to get, for closed-loop consideration:

$$
\begin{aligned}
& D=\sqrt{K M(M-1)} \\
& D_{2}=\sqrt{\frac{K M}{M-1}}
\end{aligned}
$$

Hence, (36) and (37) conventently determine dc quantities for open-loop considerations, while (38) and (39) are likewise useful for closed-loop considerations.

It is now interesting to compare the open-loop dc gain in the discontinuous conduction mode given by (36) with the corresponding dc gain in the continuous conduction mode, which, for the ideal boost converter is

$$
M=\frac{1}{1-D}
$$


Hence, the ideal dc gain (40) is dependent on duty ratio $D$ only and not on circuit parameters (such as $L, R$ ) or switching frequency $f$. In sharp contrast to this, the dc gain $M$ in the discóntinuous conduction mode (36) is dependent also on $K$ in addition to $D$ and hence is a strong function of switching frequency $f_{8}$, inductance $L$, and load R. Nevertheless, when the converter is used in this mode in a closed-loop regulator, the selfcorrecting feature of the duty ratio $D$ would compensate any possible changes of load $R$ or switching frequency $f_{s}$ and still keep the output voltage relatively constant.

Another question naturally arises in comparison of the two dc gains: when do we calculate dc gain from one (36) or the other formula (40), or, what is the criterion to determine in which of the two modes (continuous or discontinuous) the converter is operating? The answer is provided easily with reference to Fig. 5. When the second interval $D_{2} T_{8}$ is smaller than interval (1-D) $T_{s}$, the converter is operating in the discontinuous con- 8 duction mode, and in continuous mode otherwise, so the criterion becomes

continuous conduction mode

$$
\mathrm{D}_{2}>1-\mathrm{D}
$$

discontinuous conduction mode

$$
\mathrm{D}_{2}<1-\mathrm{D}
$$

To obtain a convenient quantitative measure we find, first, what happens exactly on the boundary between the two modes of converter operation, or

boundary between two conduction modes

$$
D_{2}=1-D
$$

By use of (37) in (43), the equation to determine the critical value of parameter $K$, that $1 s, K_{\text {crit }}$ for which
this happens, is

$$
\sqrt{\mathrm{K}_{\mathrm{crit}}^{2}+4 \mathrm{~K}_{\mathrm{crit}} \mathrm{D}^{2}}=2 \mathrm{DD}^{\prime}-\mathrm{K}_{\mathrm{crit}}
$$

from which

$$
\mathrm{K}_{\text {crit }}=\mathrm{DD}^{\prime 2}
$$

The solution (45) is the proper solution of (44) since $2 \mathrm{DD}^{\prime}-\mathrm{K}_{\mathrm{c}}=2 \mathrm{DD}^{\prime}-\mathrm{DD}^{\prime 2}=2 \mathrm{DD}^{\prime}\left(2-\mathrm{D}^{\prime}\right)=2 \mathrm{DD}^{\prime}(1+\mathrm{D})$ is always posfletve regardless of $D$, resulting in a proper positive right-hand side of (44). With this, the criteria (41) and (42) for determination of the operating mode become

$$
\text { continuous conduction mode } \mathrm{K}>\mathrm{K}_{\text {crit }}
$$

discontinuous conduction mode

$$
\mathrm{K}<\mathrm{K} \text { crit }
$$

boundary between two conduction modes

$$
\mathrm{K}=\mathrm{K}_{\mathrm{crit}}
$$

where $K$, as given before by (33), is a function of parameters $L, R$, and $f_{s}$, while $K_{c r i t}$ is a function of
the duty ratio $D$ on $1 y$.

We now investigate how these criteria, (46) through $(48)$, behave throughout the duty ratio range $D \in[0,1]$. To facilitate this insight, $K$ crit is plotted as a function of duty ratio $D$ in Fig. 7 . Ast seen in Fig. 7a, $K_{\text {crit }}$ (D) has a maximum of $4 / 27$ at $D=1 / 3$. This now enables an important conclusion about operating mode to be drawn. Namely, if the parameters $L, R$, and $f$ are such that the computed parameter $K$ is greater than $\frac{8}{2} 27$, expression (46) is satisfied regardless of duty ratio $D$. Hence for $K>4 / 27$ the converter always operates in the continuous conduction mode, no matter what the operating condition (duty ratio $D$ ) is. However, if parameters $L, R$, and $f_{s}$ are such that $K<4 / 27 \sim 0.15$ the situation becomes as shown in Fig. 7a, where the particular example of $K=$ $0.08<0.15$ is chosen. For a certain range of duty ratio $D$, that is $D_{10}<D<D$ (as shown by the shaded area in Fig. $7 \mathrm{a}$ ), mine condition (47) is satisfied and the converter operates in the discontinuous conduction mode, while for the remaining portions of the operating range $\left(0<D<D\right.$ and $\left.D_{1}<D<1.0\right)$ it again operates in the continuious conduction mode, since then inequality (46) holds.

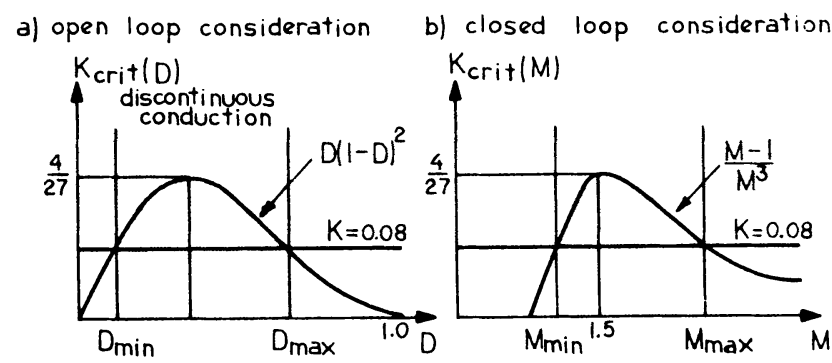

Fig. 7. Determination of the operating mode (continuous or discontinuous) for the ideal boost converter of Fig. 1.

This discussion has been in terms of open-loop considerations, when duty ratio $D$ is given and externally controlled. However, as before for dc conditions, it is desirable to have the boundary condition (45) in terms of the dc gain $M$, which is a more suitable quantity for closed-1oop considerations. This can easily be done since the dc gain $M$ is continuous across the boundary (as seen by use of (43) in (29) resulting in (40)), and thus substitution $D=(M-1) / M$ in (45) gives

$$
K_{\text {crit }}=\frac{M-1}{M^{3}}
$$

This function $\mathrm{K}_{\mathrm{crit}}(\mathrm{M})$ is plotted in Fig. $7 \mathrm{~b}$, and $\mathrm{a}$ similar discussion applies. However, now the maximum of $K$ (M) of $4 / 27$ is obtained for gain $M=1.5$. As before, for $K<4 / 27$, the converter is in the discontinuous conduction mode, but now for dc gain $M$ in the range $M_{m i n}<M<M$ as shown by the shaded area in Fig. $7 \mathrm{~b}$. ${ }^{\text {This }}$ reveals a potentially serious problem if the boost regulator were designed (and compensated) to operate in the discontinuous conduction mode only. Namely, during the initial turn-on process, the output voltage starts from zero, and the converter would have to pass through the continuous conduction region first (for $1<M<M_{\min }$ ), before coning to the discontinuous conduction region (shaded area in Fig. $7 \mathrm{~b}$ ). This would suggest possible stability problems, if the closed-loop were not compensated to assure stable operation in the continuous conduction mode as well.

From the standpoint of the dc gains (as a function of duty ratio $\mathrm{D}$ ), the situation corresponding to that of Fig. 7 is shown in Fig. 8 for some $K<4 / 27$.

From the dc gains for both conduction modes shown in Fig. 8, it becomes obvious that the actual dc gain will follow the larger of the two gains, thus the mode of operation w111 change accordingly as the duty ratio changes from 0 to $₹$. Also in the close vicinity of gain $M=1(1-M-M, n)$, the converter is always operating in the contpinuous conduction mode. Thus, the 
problem of having, for example, $D_{2}$ infinite when $M \rightarrow 1$ from (39) is only a fictitious one, since (39) is for the discontinuous conduction mode and hence not applicable in the vicinity of and at gain $M=1$.

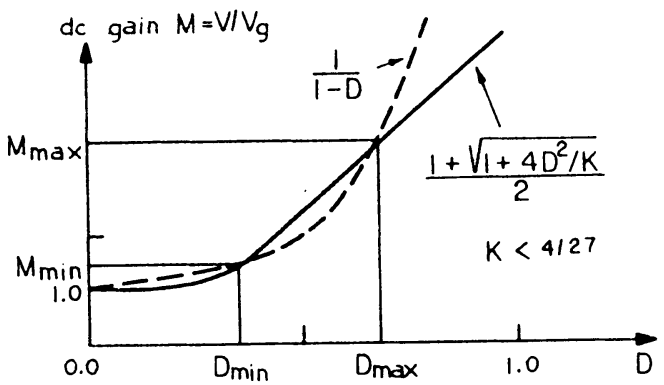

Fig. 8. Boost converter de voltage gains in continuous and discontinuous conduction modes as a function of duty ratio $D$.

We conclude this dc analysis with some numerical examples and related quantitative and qualitative significance of the dimensionless parameter $K$. For example, for the set of parameters $L=880 \mu \mathrm{H}, \mathrm{R}=220 \Omega$ and $\mathrm{f}=$ $20 \mathrm{kHz}$, we compute $K=2 \mathrm{Lf} / \mathrm{R}=0.16$. Therefore, since $K=0.16>4 / 27$, the converter will with this set of parameters always operate in the continuous conduction mode. However if, for example, the switching frequency is reduced to $f_{s}=10 \mathrm{kHz}$, this results in $\mathrm{K}=0.08<4 / 27$ and some range of discontinuous conduction operation should be expected (see Figs. 7 and 8). Therefore, the reduction of parameter $K$ below $4 / 27$ causes this transition. From the definition of $K$ in (33) this reduction and change to the discontinuous conduction mode is qualitatively achieved by three means: increase of load $R$, decrease of the inductance $L$ or suitching frequency $f_{s}$. There is also a fourth way to enter the discontinuous conduction mode, and that is to change the operating condition, the duty ratio $D$, as 1llustrated in Fig. 7 and Fig. 8, but only if the condition $K<4 / 27$ is met.

Very often, however, out of all these four possibilities, one is mostly interested in how the change of load $R$ affects the operating mode. Namely, the parameters $L$ and $f_{s}$ are usually design parameters whose choice may depend on the size and efficiency requirements of the converter or regulator. On the other hand, the range of variation of duty ratio $D$, or equivalently of gain $M$, is a design requirement in a closed-loop implementation since the output voltage $\mathrm{V}$ is maintained constant against the range of variation of input voltage $V$ (hence range of $M=V / V$ ) by the action of negative fedback. The load $R$ also can have a wide range of change depending on the user of the regulator, and is often out of the designer's control. Hence, determination of the converter operating mode with respect to changes of load $R$ becomes important. This can be easily accomplished by finding an equivalent of (45) and (49) respectively, as

$$
\begin{aligned}
& R_{\text {crit }}=\frac{1}{D^{\prime 2}} R_{\text {nom }} \\
& R_{\text {crit }}=\frac{M^{3}}{M-1} R_{\text {nom }}
\end{aligned}
$$

where $R_{\text {nom }}$ is a design parameter defined by

$$
\mathrm{R}_{\text {nom }} \triangleq 2 \mathrm{Lf}_{\mathrm{s}}
$$

The criteria for determination of the operating mode, (46), (47), and (48), then become continuous conduction mode

$$
R<R_{\text {crit }}
$$

discontinuous conduction mode

$$
R>R_{\text {crit }}
$$

boundary between two modes

$$
R=R_{\text {crit }}
$$

Let us now 1llustrate this on a numerical example. For $L=880 \mu \mathrm{F}, \mathrm{f}_{\mathrm{f}}=20 \mathrm{kHz}$ we calculate $\mathrm{R}=35.2 \Omega$. By the same argument as before (see F1gs. $7^{\text {nomd }}$ and, for example), the converter will always operate in the continuous conduction mode if

$$
\mathrm{R}<\frac{27}{4} \mathrm{R}_{\text {nom }}
$$

or for the given numerical example for $R<238 \Omega$. When $R>238 \Omega$ there will be a range of gain M (see Fig. 8) for which the converter operates in the discontinuous conduction mode.

This concludes the extensive dc analysis and we now turn to the dynamic (ac small-signal) model analysis of this ideal boost converter example.

Dynamic (ac small-signal) model analysis

Before we apply the general result to this 1 deal boost converter example, let us first put the constraint (27) into a more suitable form by using the steady-state average inductor current I of (31) to get

$$
i=\frac{v_{g} d T_{s}}{2 L}=\frac{v_{g} d}{v_{g} D} I
$$

By use of perturbation equation (57), model description (26) and definition (12) in the general result given by (22) through (24), we obtain

dyaamic (ac small-s1gnal) model

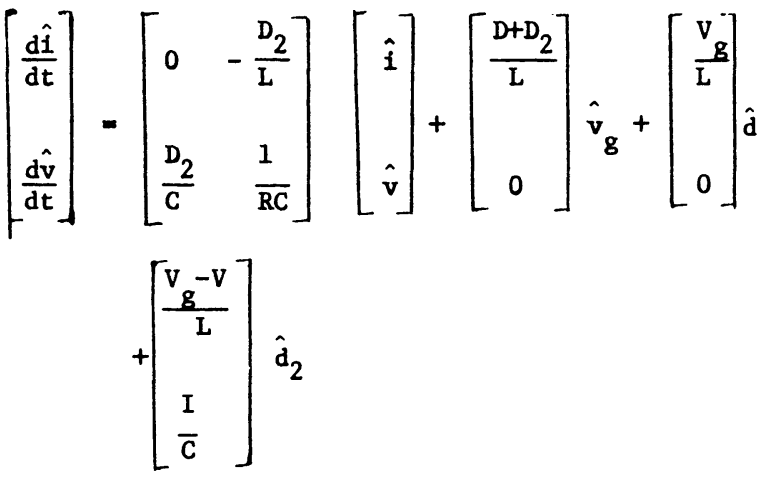

with additional constraints

$$
\begin{aligned}
& \frac{\hat{d} I}{d t}=0 \\
& \hat{i}=\frac{I}{V_{g}} \hat{v}_{g}+\frac{I}{D} \hat{d}
\end{aligned}
$$

As opposed to the general result, we can now for this specific example enter the constraints (59) and (60) into dynamic model description (58). The introduction of (59) reduces the first dynamic equation in (58) to a static one, and after proportionality constant $L$ is removed the dynamic mode1 becomes 


$$
\begin{aligned}
0 & =-D_{2} \hat{v}+\left(D+D_{2}\right) \hat{v}_{g}+v_{g} \hat{d}+\left(v_{g}-V\right) \hat{d}_{2} \\
c \frac{d \hat{v}}{d t} & =D_{2} \hat{i}-\hat{v} / R+I \hat{d}_{2}
\end{aligned}
$$

with additional constraint (60). Note, however, that now the first static equation (61) actually determines the unknown modulation quantity $\mathrm{d}_{2}$ (modulation of the second

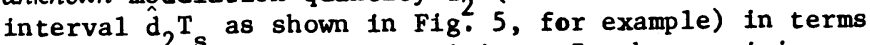
of the other $\mathrm{dc}$ and ac quantities. In the remaining dynamic equation $(62)$, besides this modulation $\hat{d}_{2}$ which we can now express from (61), current modulation $\hat{i}$ also appears. But, from the perturbation equation $I(60)$ it is also determined in terms of the known ac quantities (forced modulations $\hat{v}$ and $\hat{d}$ ). In general, both equations $(60)$ and $(61)$ could have both modulation quantities $\hat{i}$ and $\hat{d}_{2}$ for some arbitrary converter. But, they are linear algebraic equations and could be solved for $\hat{i}$ and $\hat{d}_{2}$ in terms of other ac quantities and then substituted in the remaining dynamic description (which could be, for some converter with more than two storage elements, higher than the first order model given by (62)).

Another general feature, which is hidden in this model, is that (61) can be considered as a consequence of the equation

$$
\left(d+d_{2}\right) v_{8}=d_{2} v
$$

which after usual perturbation and linearization steps and subtraction of dc terms reduces to (61). Hence, in analogy to (57), equation (63) can now be designated perturbation equation II. The appearance of (63) in the modelling will become more apparent later in the hybrid modeliing and circuit averaging techniquess. But in any case, the unknown modulation quantities 1 and $d_{2}$ come as the solution of two linear algebraic equations, which are essentially linearized versions of perturbation equations $I$ and $I I,(57)$ and (63) respectively.

To complete the dynamic model description we simply substitute $(60)$ and the solution of $\hat{d}_{2}$ from (61) in (62) to get

$C \frac{d \hat{v}}{d t}=-\left(\frac{D_{2} I}{V-V_{g}}+\frac{1}{R}\right) \hat{v}+\left(\frac{D_{2}}{V_{g}}+\frac{D+D_{2}}{V-V_{g}}\right) I \hat{v}_{g}+\left(\frac{D_{2}}{D}+\frac{v_{g}}{V-V_{g}}\right) I \hat{d}$

Since this dynamic model has significance only for the closed-1oop regulator, it is convenient to express all dc quantities in terms of $M, K, R$ and output voltage $V$, as was explained before in the dc analysis. Hence by use of (38), (39) and (30) we obtain

$$
c \frac{d \hat{v}}{d t}=-\frac{2 M-1}{M-1} \frac{1}{R} \hat{v}+\frac{M}{M-1} \frac{2 M-1}{R} \hat{v}_{g}+\frac{2 V}{R} \frac{1}{\sqrt{K M(M-1)}} \hat{d}
$$

In (65) all proportionality constants would become infinite and meaningless when $M=1$. However, it was explained in the dc analysis that in the vicinity of and at gain $M=1$, the boost converter always operates in the continuous conduction mode, hence a different dynamic mode1 applies.

Is is now easy to obtain from (65) two transfer functions of interest

$$
\begin{aligned}
& G_{v g}=\frac{\hat{v}(s)}{\hat{v}_{g}(s)}=G_{\text {og }} \frac{1}{1+s / \omega_{p}} \\
& G_{v d}=\frac{\hat{v}(s)}{\hat{d}(s)}=G_{\text {od }} \frac{1}{1+s / \omega_{p}}
\end{aligned}
$$

where

$$
\omega_{p}=\frac{2 M-1}{M-1} \frac{1}{R C}
$$

and

$$
G_{o g}=M, \quad G_{o d}=\frac{2 V}{2 M-1} \sqrt{\frac{K M}{M-1}}
$$

As seen from (66) both transfer functions have a single pole $\omega$ and no zeros. This is qualitatively completely diffefent dynamic behavior than in the continuous conduction mode where two poles and even a right half-plane zero are obtained [2] (for the $G$ transfer function only). This in turn suggests easier compensation (even no compensation at a11) and reduced stability problems if the converter as a part of a switching regulator is operating consistently in the discontinuous conduction mode. But, a potent1al danger exists there: any significant transient changes (such as sudden change of input voltage or temporary substantial change of load R) could move the operating point to the continuous conduction region (see Fig. 8) and cause instability. Another problem is inherent to the discontinuous conduction mode. In addition to the output current, now the input current becomes pulsating as well (as shown in Fig. 5) which increases electromagnetic interference problems. Hence, a decision on the cholce of operating mode becomes a complex one, depending on the particular design requirements. To facilitate that decision, we now undertake the task of developing useful circuit models of the switching converter operating in the discontinuous conduction mode.

\section{HYBRID MODELLING IN DISCONTINUOUS CONDUCTION MODE}

We demonstrate in this section how for any specific converter a useful circuit model of the basic statespace averaged model ( 8 ) can be found, appropriately modified by inclusion of the constraint ( 9 ), and supplemented by the additional constraint (10). In terms of the Flowchart of Fig. 4 we will proceed from block $2 a$ through $2 c$ to arrive at the circuit model in block $2 b$. Again this is 1llustrated on the same 1deal boost converter example as in the previous section.

When the boost converter description (26) and (27) is applied to (8), (9) and (10) the following basic state-space averaged model results:

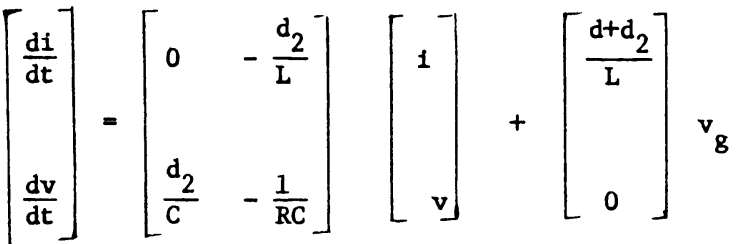

with additional constraints

$$
\begin{aligned}
& \frac{d i}{d t}=0 \\
& i=\frac{v_{d} d T s}{2 L}
\end{aligned}
$$

It now becomes clear that introduction of (70) into (69) reduces the first dynamic equation to perturbation equation II as given before by (63). But, instead of introducing this substitution, let us first find the circuit realization of the state-space equations (69) as shown in Fig. 9. 
The constraint (70) leads, in the circult model of Fig. 9, to effective disappearance of the inductance $L$, since $v_{1}=L d 1 / d t=0$. The resulting equality of the two voltage generators produces again the perturbation equation II given by (63). At the same time shorting of the inductance causes reduction of system order by one, and effectively a single pole transfer function result becomes apparent.

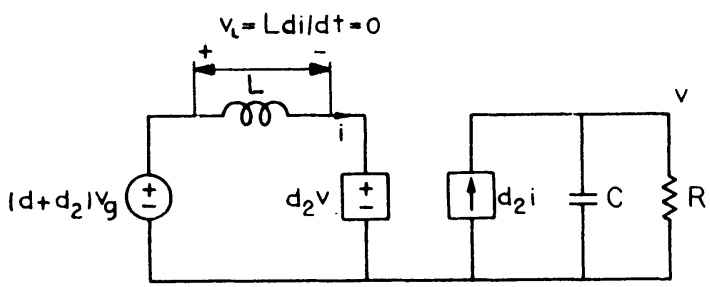

Fig. 9. Circuit realization of the state-space model (69), with constraint (70) also included.

Let us now put the circult of Fig. 9 into more elegant form, by introducing a dc and ac transformer in place of the two dependent generators in Fig. 9. Also it is desirable to have source voltage $v$ effectively at the input of the converter, rather than as some modified quantity as $\left(d+d_{2}\right) v_{\text {in Fig. }}$. However, this is easily accomplished by infroduction of another dc and ac transformer at the input of the converter. In addition, the true input current into the converter becomes properly exposed as seen in the basic circuitaveraged model of $\mathrm{Fig}$. 10. In addition to the circuit model in Fig. 10 we need the remaining constraint (71) to complete the description of the converter in discontinuous conduction mode (as also displayed in Fig. 10). As before, the circuit model and the additional perturbation equation are valid for both dc and ac conditions. Hence the two transformers in Fig. 10 are operating both at ac and dc and the appropriate symbol is introduced.

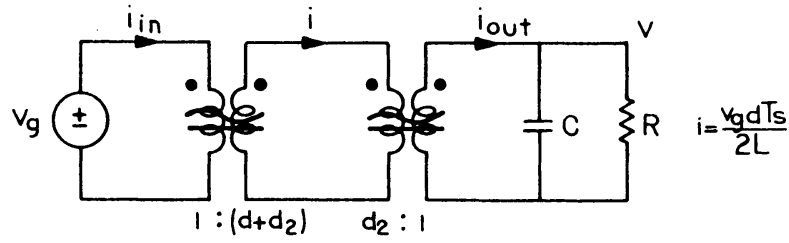

Fig. 10. Basic circuit averaged model for the ideal boost converter in the discontinuous conduction mode.

A word about the new transformer symbol introduced in Fig. 10 is appropriate here. In the modelling of dc-to-dc converters a need naturally arises to have as a convenient modelling tool spectal types of transformers: a transformer which operates for both ac and dc signals, as for example that in Fig. 10, and also a transformer which only works at dc (for which the need will arise later in Section 5.1). Even though these transformers are not physically realizable they are, nevertheless, useful in modelling the basic converter function: dc-to-dc conversion. Hence, as an indicator of their specific functions, the symbols of Fig. 11 are intro-

a) dc and ac transformer b/dc transformer

c) ac transformer
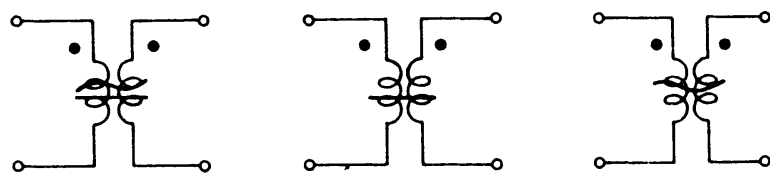

duced. For consistency, the conventional, physically realizable, ac transformer only, is pictorlally represented as in Fig. 11c. Later in Section 5.2, for similar purposes, the same overprint glyphs will be used with resistance symbols.

Following the procedure outlined in this section one can easily obtain the basic averaged circuit models of the three common power stages of Fig. 1. These models for discontinuous conduction mode are summarized in Fig. 12.

a) buck power stage:

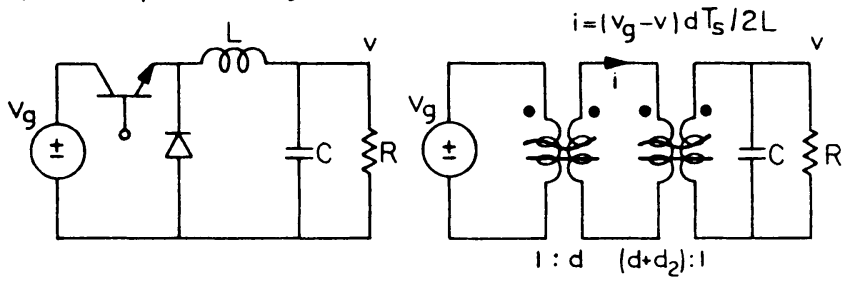

b) boost power stage:

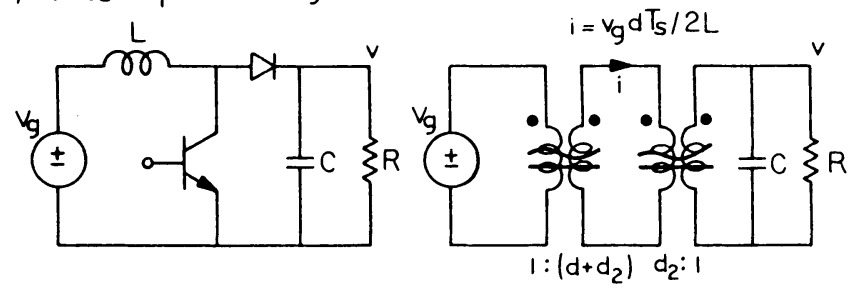

c) buck-boost power stage:

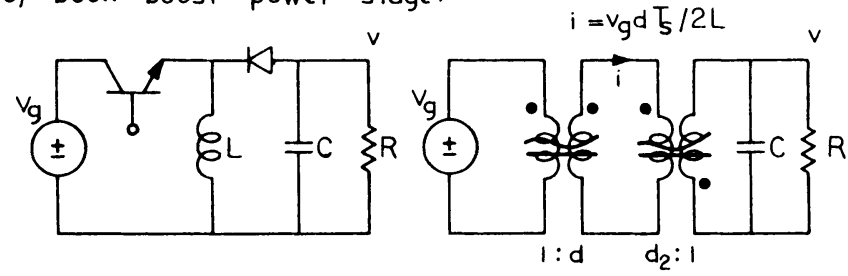

Fig. 12. Summary of the basic circuit averaged models for three common power stages in discontinuous conduction mode.

5 CIRCUIT AVERAGING IN DISCONTINUOUS CONDUCTION MODE

In this section the alternative path $b$ in the Flowchart of Fig. 4 is followed and the perturbation and linearization steps corresponding to those in statespace averaging path $a$ are applled to the circult model to arrive at the final circuit averaged models, separately for steady-state (dc) and dynamic (ac) response.

We continue with the same ideal boost converter example and hence use as a starting model the circuit model of Fig. 10. Even though that circuit model was obtained by following hybrid modelling, we emphasize also the other possibility. Namely, it could have been obtained directly by averaging the three switched circuit models of Fig. 6 using the standard circuit averaging technique and supplementing it by the appropriate constraints (70) and (71).

\section{Perturbation}

If the averaged circuit model of Fig. 10 is perturbed together with its perturbation equation I according to

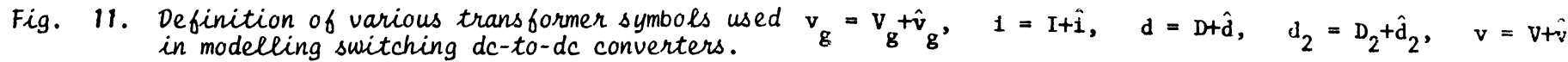


the nonlinear model of Fig. 13 results.

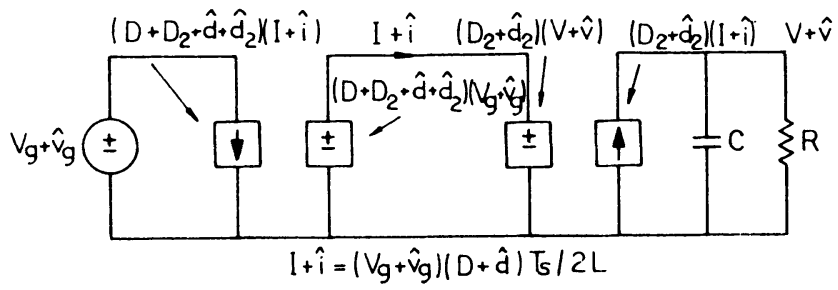

Fig. 13. Perturbation of the basic averaged circuit model in Fig. 10 results in this nonlinear circuit model.

\section{Linearization} that is

With the small-signal assumption on perturbation,

$\hat{\mathrm{d}} \ll \mathrm{D}, \quad \hat{\mathrm{d}}_{2} \ll \mathrm{D}_{2}, \quad \hat{\mathrm{i}} \ll \mathrm{I}, \quad \hat{\mathrm{v}} \ll \mathrm{V}, \quad \hat{\mathrm{v}}_{\mathbf{g}} \ll \mathrm{V}_{\mathrm{g}}$

the second-order terms in F1g. 13 can be neglected and the linearized model of Fig. 14 obtained.

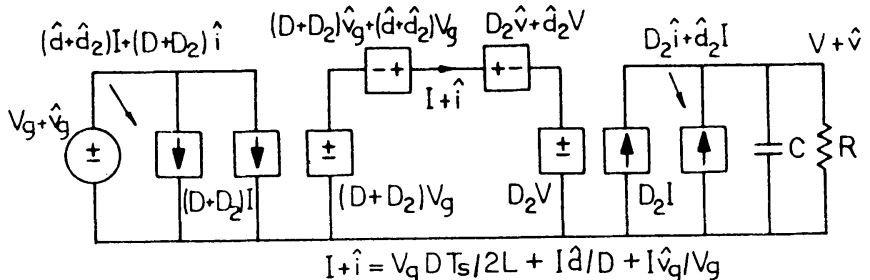

Fig. 14. Model of Fig. 13 linearized to include dc and ac small-signal models.

The circuit model in Fig. 14 together with the dc and ac part of the perturbation equation I (also shown in Fig. 14) completely determines both models. At this point, we continue to develop separately the two circuit models -- the steady-state (dc) circuit model and the dynamic (ac sma11-signal) model.

\subsection{Steady-state (dc) circuit mode1}

With all ac quantities set to zero, the dc circuit model is obtained directly from F1g. 14, and upon substitution of dc dependent generators by the dc transformer symbols, the circuit model in Fig. 15 results.

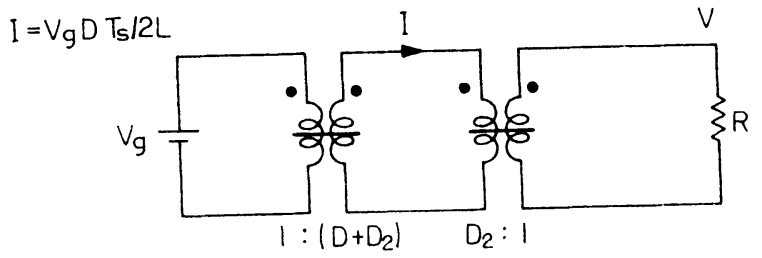

Fig. 15. Final dc circuit model for the boost converter in the discontinuous conduction mode.

This circuit model is also supplemented by the dc part of the perturbation equation $I$, which is, of course, the same as (31). From the circult model in Fig. 15 the other two dc relations (29) and (30) are obtained. Hence the dc circuit model leads to the same dc conditions and results discussed at length in Section 3.1 on state-space averaging.

We now turn to the development of the dynamic (ac) circuit model.

\section{$\underline{5.2}$ Dynamic (ac) circult model}

After the steady-state (dc) quantities are subtracted from the circuit model in Fig. 14 (and perturbation equation as well) the ac circuit model in Fig. 16 is obtained.

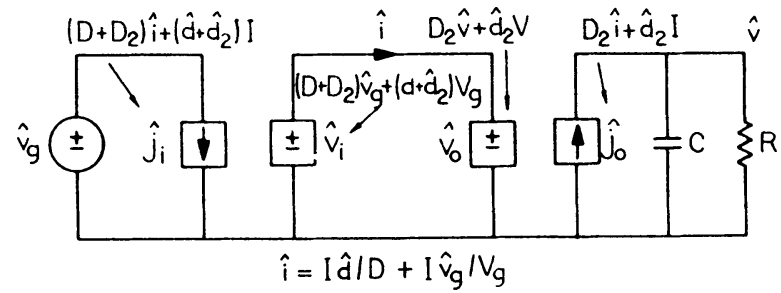

Fig. 16. Dynamic lac small-signal) circuit model for the boost converter with the constraint on modulation $i$ (perturbation equation I) not yet included in the circuit model.

From Fig. 16 it is obvious that the two dependent current generators are functions of two yet undetermined modulation quantities $\hat{d}_{2}$ and $\hat{i}$, since the other quantities are efther already determined from the $d c$ circult model (such as $D_{2}, I$ ) or are known driving quantities (as $D$ and $\hat{d}$ ). While the current modulation is already avallable through the linearized perturbation equation I (see Fig. 16), the other modulation quantity $\hat{\mathrm{d}}_{2}$ can easily be obtained from the inside loop of Fig. 16. Namely, since the two voltage generators in Fig. 16 must be equal, we get

$$
\left(D+D_{2}\right) \hat{v}_{g}+\left(d+\hat{d}_{2}\right) v_{g}=D_{2} \hat{v}+\hat{d}_{2} v
$$

Note that this is the same equation as the first (static) equation (61) of the state-space averaged model. Now it is easy to see that (74) and (61) came out actually as a consequence of the perturbation and linearization steps applied to the perturbation equation II (63), since the voltage generators in Fig. 16 resulted from the perturbation and linearization of the voltage generators in Fig. 9, which have been shown to be equal for discontinuous conduction mode (owing to $\mathrm{di} / \mathrm{dt}=0$ constratint).

The equation (74) can now be solved for the unknown modulation $\mathrm{a}_{2}$ and, together with the perturbation equation defining $\hat{i}$, determines the two current generators in terms of the known modulation quantities as follows:

$$
\begin{aligned}
& \hat{j}_{1}=\left(\hat{d}_{+} \hat{d}_{2}\right) I+\left(D+D_{2}\right) \hat{i}=\frac{2 v I}{V-v_{g}} \hat{d}+\frac{V}{v_{g}} \frac{\left(D+D_{2}\right) I}{V-v_{g}} \hat{v}_{g}-\frac{D_{2} I}{V-v_{g}} \hat{v} \\
& \hat{j}_{0}=\hat{d}_{2} I+D_{2} \hat{i}=\frac{2 v_{g} I}{V-v_{g}} \hat{d}+\frac{V}{v_{g}} \frac{2 V-v_{g}}{V-v_{g}} \frac{1}{R} \hat{v}_{g}-\frac{V}{V-v_{g}} \frac{1}{R} \hat{v}
\end{aligned}
$$

Since the converter dynamic model is usually used in closed-loop regulator applications, we conveniently express all dc quantities in terms of $M, K, R$ and output regulated voltage $V$ (as explained before) to arrive at

$$
\begin{aligned}
& j_{1}=\frac{2 V}{R} \sqrt{\frac{M}{K(M-1)}} \hat{d}+\frac{M^{3}}{M-1} \frac{1}{R} \hat{v}_{g}-\frac{M}{M-1} \frac{1}{R} \hat{v} \\
& \hat{j}_{0}=\frac{2 V}{R} \frac{1}{\sqrt{K M(M-1)}} \hat{d}+\frac{M(2 M-1)}{M-1} \frac{1}{R} \hat{v}_{g}-\frac{M}{M-1} \frac{1}{R} \hat{v}
\end{aligned}
$$

By use of (77) and (78) in the circuit model of Fig. 16, the circuit model in Fig. 17 is generated. 


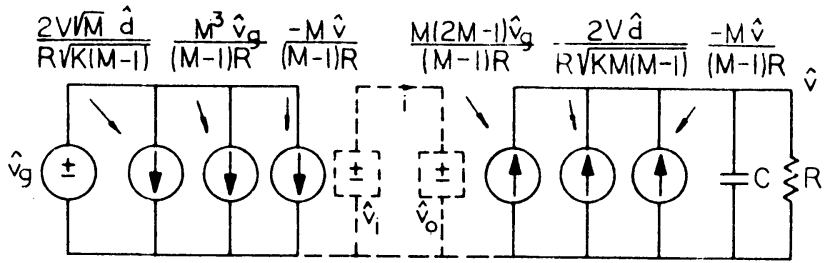

Fig. 17. Dynamic (ac small-signal) circuit model of the boost converter with perturbation equation I (for modulation $i$ ) and perturbation equation II lequality of the voltage generators $\hat{v}_{i}$ and $\hat{v}_{0}$ ) included in the circuit model.

The two voltage generators $\hat{v}_{i}$ and $\hat{v}_{0}$ in Fig. 17 are purposely shown in dotted lines to emphasize the fact that they are no longer essential, since the information provided by them (74) has already been used to find modulation $\hat{d}_{2}$ and substituted elsewhere in the circuit model. Therefore they can now be omitted from the circuit model. Finally, by modelling the current generators in Fig. 17 which are proportional to voltages across them as ac resistors only, the final circuit model of Fig. 18 is obtained.

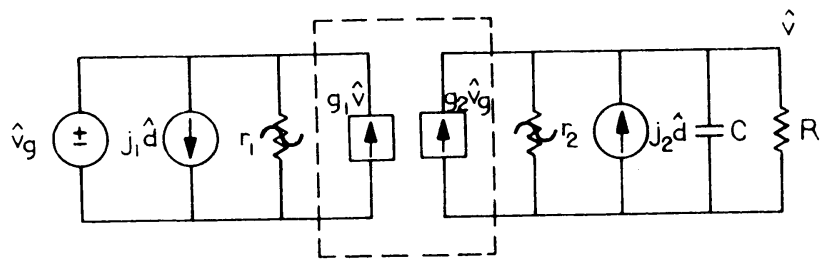

Fig. 18. Final ac small-signal circuit model for boost converter in the discontinuous conduction mode.

The element values in Fig. 18 are defined as

$$
\begin{array}{ll}
j_{1}=\frac{2 V}{R} \sqrt{\frac{M}{K(M-1)}}, \quad r_{1}=\frac{M-1}{M^{3}} R, \quad g_{1}=\frac{M}{M-1} \frac{1}{R} \\
j_{2}=\frac{2 V}{R} \sqrt{\frac{1}{K M(M-1)}}, \quad r_{2}=\frac{M-1}{M} R, \quad g_{2}=\frac{M(2 M-1)}{M-1} \frac{1}{R}
\end{array}
$$

Also since $r_{1}$ and $r_{2}$ are ac resistances only, the appropriate symbol consistent with that adopted for the ideal transformer designation (see Fig. 11, for example) is used in Fig. 18. The two current generators inside the dotted-1ine box in Fig. 18 are used with square symbols to emphasize the fact that they are dependent current generators (on some other quantities in the circuit).

From the circuit model in Fig. 18 and by use of element definitions (79) and (80), the two transfer functions $G$ and $G$ can be derived. It can easily be verified that they agree exactly with those obtained before, $((66),(67)$ and (68)), using state-space averaging. An interesting observation with regard to the topology of the circuit model in F1g. 18 can be made. Namely, to arrive at these two transfer functions, only the elements in the output port $j_{2}, r_{2}$ and $g_{2}$ have been used, without any need for input port description. However, the input port description becomes mandatory if the determination of the complete circuit model is desired, since it properly models the fmportant input properties (both open- and closed-loop input impedances, for example), as will be illustrated in Section 7.2. Moreover, the output port model now does affect the input properties through the dependent current generator $\mathrm{g}_{1} \mathrm{v}$ in Fig. 18 .
An interesting comparison with the circult model topologies for the continuous conduction mode $[1,2]$ seems appropriate here. While in the continuous conduction mode the effect of duty ratio modulation $\hat{d}$ was expressed through duty ratio dependent voltage and current generators, here two duty ratio dependent current generators (one at the input and the other at the output port) appropriately account for both input and transfer properties (and output properties, as well). Another distinction and unique feature of the circuit model of Fig. 18 is the presence of ac resistances only (which are in general dependent on an operating condition, the gain $M$ ), a characteristic not present in the continuous conduction mode. But despite these topological and qualitative differences, the circuit models for continuous conduction mode $[1,2]$ and discontinuous conduction mode (Fig. 18) have something very important in common: they both represent a complete linearized circuit model which accurately represents not only transfer properties but input and output properties as well.

The method outlined in this section, and fllustrated for the boost converter, is applied to the other two converters of Fig. 1 and results are presented in various tabular forms (including the boost circuit example) in Section 6 on a canonical circuit model.

\section{CANONICAL CIRCUIT MODEL FOR DISCONTINUOUS} CONDUCTION MODE

In this section the canonical circuit model for discontinuous conduction mode (block 5 in the Flowchart of Fig. 4 or Fig. 18) is obtained for the three common switching converters of Fig. 1 , and thanks to its fixed circuit topology, the results are conveniently summarized in the form of various tables, separately for dc and for ac small-signal circuit models.

From the dc conditions and by following the derivations presented in Section 3.1, the simple formulas for determination of the boundary between the two conduction modes may also be found for the buck and buckboost converters. These results, analogous to (45) and (49) through (51) for the boost converter, are again tabulated for all three common converters of Fig. 1. This then ultimately determines which of the circuit models (those of [2] or those of Sections 5.1 and 5.2) should be chosen for given parameter values and operating conditions of a closed-loop switching regulator. An interesting pictorial interpretation facilitating this decision is given in terms of the frequency scale and position of another "Inherent"frequency $\omega_{0}$ (frequency defined by converter element values, like $\omega$ and $f_{c}$ before) with respect to switching frequency ${ }_{f_{s}}$.

Finally, both dc and ac transfer properties are experimentally verified on a particular buck-boost converter breadboard and excellent agreement with the predictions is observed, thus confirming the high accuracy of the circuit models for the discontinuous conduction mode.

\subsection{Derivation of the canonical circuit models for discontinuous conduction mode}

In this section the canonical circuit models (both dc and ac small-signal circuit models) for the two remalning converters of Fig. 1 are derived from the basic circuit averaged models in Fig. 12 .

Buck converter in discontinuous conduction mode

With regard to the dc circuit model derivation, a ceneral observation seems appropriate here. Namely, the dc circult model of the boost converter (F1g. 15) could have been obtained directly from the unperturbed cir- 
cult model in Fig. 12b by simply taking all quantities to be dc quantities and as usual considering the capacitance $C$ to be open for dc signals. Hence, as should have been expected, the circuit models in Fig. 12 together with the additional expressions for the average inductor current 1 are valid de models. But this is exactly why it was previously emphasized that the presented methods for finding dc and ac models are consistent with each other. After all, ac small-signal models really represent the linearized perturbation around some steady-state (dc) conditions. Hence, by perturbation and linearization of the circuit models in Fig. 12, the ac circuit models consistent with the superimposed dc circuit models result. Therefore, the dc circuit model for the buck converter is as in Fig. 12a with dc quantities $d=D, d_{2}=D_{2}, 1=I, v_{g}=v_{g}, v=V$ and $d c$ transformers only.

After usual perturbation and linearization steps are applied to the circuit model of Fig. 12a, the dynamic (ac) circuit model in Fig. 19 is obtained.

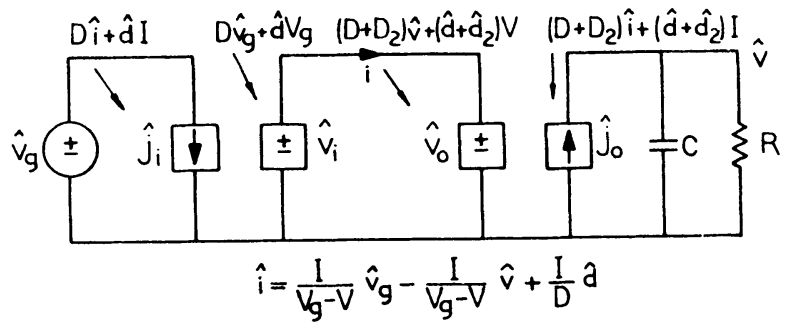

Fig. 19. Dynamic (ac small-signal) circuit model for the buck converter in discontinuous conduction mode with corresponding perturbation equation I for modulation $i$.

The perturbation equation I is different from that for the boost converter and is

$$
I=\frac{\left(v_{g}-v\right) d T_{s}}{2 L}=\frac{\left(v_{g}-v\right) d}{\left(v_{g}^{-V) D} I\right.}
$$

After perturbation and linearization of (81) we get

$$
\hat{i}=\frac{I}{V_{g}-V} \hat{v}_{g}+\frac{I}{D} \hat{d}-\frac{I}{V_{g}-V} \hat{v}
$$

When the unknown modulation quantity $\hat{d}_{2}$ is found from equality of the two voltage generators in F1g. 19, and by use of (82), the two current generators in F1g. 19, after expression of dc quantities in terms of closedloop parameters $M, K, R$, and $V$, become

$$
\hat{j}_{1}=j_{1} \hat{d}+\hat{v}_{g} / r_{1}-g_{1} \hat{v} ; \quad \hat{j}_{0}=j_{2} \hat{d}+g_{2} \hat{v}_{g}-\hat{v} / r_{2}
$$

where

$$
\begin{aligned}
& f_{1}=\frac{2 V}{R} \sqrt{\frac{1-M}{K}}, \quad r_{1}=\frac{1-M}{M^{2}} R, \quad g_{1}=\frac{M^{2}}{1-M} \frac{1}{R} \\
& f_{2}=\frac{2 V}{R} \frac{1}{M} \sqrt{\frac{1-M}{K}}, \quad r_{2}=(1-M) R, \quad g_{2}=\frac{M(2-M)}{1-M} \frac{1}{R}
\end{aligned}
$$

Hence the same topology of the dynamic (ac) model for the boost converter shown in Fig. 18 is also obtained for the buck converter. In the discontinuous conduction mode, but with the model element values defined by (84) and $(85)$.
Buck-boost converter in the discontinuous conduction mode

The dc circult model for the buck-boost converter is obtained directly from the circuit model in Fig. 12c. After perturbation and linearization of the model, the dynamic (ac) c1rcuit model in Fig. 20 is obtained.

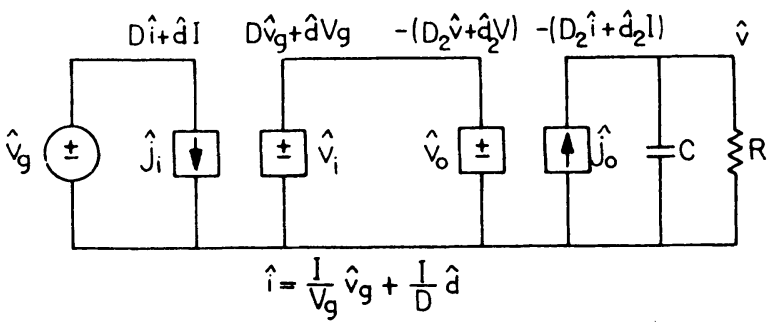

Fig. 20. Dynamic (ac small-signal) circuit model for the buck-boost converter in discontinuous conduction mode with perturbation equation I (for i) shown explicitly.

The perturbation equation $I$ is now the same as for the boost converter (71), and the two current generators $\hat{J}_{1}$ and $\hat{\jmath}_{\text {in }} \mathrm{F} 1 \mathrm{~g} .20$ are as defined in (83) but with the following element values for the buck-boost converter:

$f_{1}=\frac{2|V|}{\sqrt{\mathrm{KR}}}, \quad \mathrm{r}_{1}=\frac{\mathrm{R}}{\mathrm{M}^{2}}, \quad \mathrm{~g}_{1}=0$

$\mathrm{J}_{2}=2 \frac{|\mathrm{V}|}{\sqrt{\mathrm{K} R}} \frac{1}{M}, \quad \mathrm{r}_{2}=\mathrm{R}, \quad \mathrm{g}_{2}=\frac{2 \mathrm{M}}{\mathrm{R}}$

Again the same circuit topology of Fig. 18 results, but with element values (86) and (87). However, there is a small distinction from the previous two models since now, as seen in $(86), g_{1}=0$. Therefore there is no feedback effect from the output port to the input c1rcuit model as in the other two converters, and the openloop input impedance ts just $r_{1}$. But, this is reasonable to expect for the buck-boost converter, since it is the only converter in which the energy transferring inductance is present either solely in the input circuit (interval DT ) or solely in the output circuit (interval $D_{2} T_{s}$ ). In the other two converters (buck and boost), on the other hand, the output circuit (including $C$ and $R$ ) is at least for a portion of period $T$ connected to the input and represents a loading effect on 1t. Hence the feedback action through current generator $8_{1} \hat{v}$ is to be expected in these two converters.

The results for all three converters (buck, boost and buck-boost) are summarized in the next section.

\subsection{Summary of the canonical circuit mode1 results for three common converters}

In this section the results for both dc and dynamic (ac) canonical circuit models for buck, boost, and buckboost converter are summarized and, owing to the fixed circult model topology, convenlently listed in several tables.

In Fig. 21 the polarity of the second transformer $1: M_{18}$ inverting for the buck-boost converter and otherwise as shown. The parameters in the dc circuit model of Fig. 21 are defined in the first three columns of Table $I$, while the remaining two colums tabulate the dc relations derived from this circult model. Note, however, that this circult model can be used to determine other dc quantities as well, such as the dc input durrent $I_{\text {in }}$ in terms of the defining parameters. 


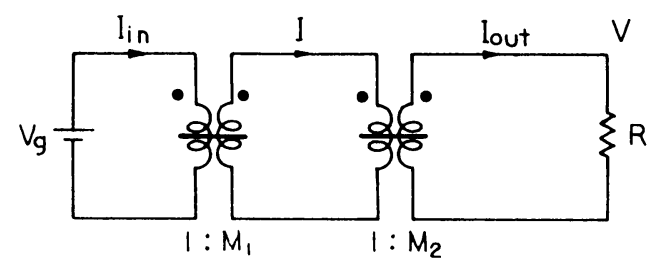

Fig. 21. Steady-state (dc) circuit model for the converters of Fig. 1 in the discontinuous conduction mode.

\begin{tabular}{|c|c|c|c|c|c|}
\hline \multirow{2}{*}{$\begin{array}{c}\text { converter } \\
\text { type }\end{array}$} & \multicolumn{2}{|c|}{ definition of dc model } & \multicolumn{2}{c|}{ derived quantities } \\
\cline { 2 - 6 } buck & $D$ & $\frac{1}{D+D_{2}}$ & $\frac{\left(V_{g}-V\right) D T s}{2 L}$ & $\frac{V}{\left(D+D_{2}\right) R}$ & $\frac{D}{D+D_{2}}$ \\
\hline boost & $D+D_{2}$ & $\frac{1}{D_{2}}$ & $\frac{V_{g} D T s}{2 L}$ & $\frac{V}{D_{2} R}$ & $\frac{D+D_{2}}{D_{2}}$ \\
\hline $\begin{array}{c}\text { buck- } \\
\text { boost }\end{array}$ & $D$ & $\frac{1}{D_{2}}$ & $\frac{V_{g} D T_{s}}{2 L}$ & $\frac{V}{D_{2} R}$ & $\frac{D}{D_{2}}$ \\
\hline
\end{tabular}

TABLE I. Definition of the dc circuit model in Fig. 21 for the three common converters of Fig. 1 operating in the discontinuous conduction mode.

\begin{tabular}{|c|c|c|c|c|}
\hline \multirow{2}{*}{$\begin{array}{c}\text { converter } \\
\text { type }\end{array}$} & \multicolumn{2}{|c|}{ open-loop consideration } & \multicolumn{2}{c|}{ closed-loop consideration } \\
\cline { 2 - 5 } buck & $\frac{2}{1+\sqrt{1+4 K I D^{2}}}$ & $\frac{K}{D} \frac{2}{1+\sqrt{1+4 K / D^{2}}}$ & $\sqrt{\frac{K M^{2}}{1-M}}$ & $K(1-M)$ \\
\hline boost & $\frac{1+\sqrt{1+4 D^{2} / K}}{2}$ & $\frac{K}{D} \frac{1+\sqrt{1+4 D^{2} / K}}{2}$ & $\sqrt{K M(M-1)}$ & $\sqrt{\frac{K M}{M-1}}$ \\
\hline $\begin{array}{c}\text { buck- } \\
\text { boost }\end{array}$ & $\frac{D}{\sqrt{K}}$ & $\sqrt{K}$ & $M \sqrt{K}$ & $\sqrt{K}$ \\
\hline
\end{tabular}

TABLE II. Summary of dc transfer properties of the three common converters of Fig. 1 in the discontinuous conduction mode expressed for open-loop as well as for closed-loop considerations.

With use now of the last three columns of Table I and the procedures outlined in Section 3 , the very useful Table II can be generated, in which the dimensionless parameter $\mathrm{K}$ is defined as before with $\mathrm{K}=2 \mathrm{~L} / \mathrm{RT} \mathrm{s}_{\mathrm{s}}=2 \mathrm{Lf} \mathrm{f}_{\mathrm{s}} / \mathrm{R}$

The element values of the dynamic (ac) circuit model in Fig. 22 for the three converters are shown in Table III.

Again, as Table II was generated from Table $I$ and only input-output dc transfer properties obtained, we can similarly generate from.Table III another, Table IV, in which only input-output ac transfer properties (transfer functions $G_{v g}$ and $G_{v d}$ ) are listed for the three converters.

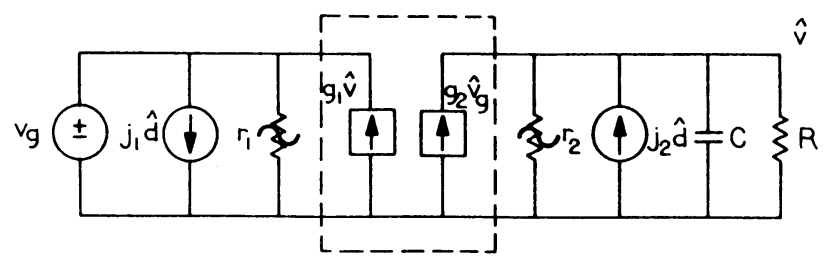

Fig. 12. Final ac small-signal circuit model for converters of Fig. 1 in the discontinuous conduction mode.

\begin{tabular}{|c|c|c|c|c|c|c|}
\hline type & $j_{1}$ & $r_{1}$ & $g_{1}$ & $j_{2}$ & $r_{2}$ & $g_{2}$ \\
\hline buck & $\frac{2 V}{R} \sqrt{\frac{1-M}{K}}$ & $\frac{1-M}{M^{2}} R$ & $\frac{M^{2}}{1-M} \frac{1}{R}$ & $\frac{2 V}{R M} \sqrt{\frac{1-M}{K}}$ & $(1-M) R$ & $\frac{M(2-M) !}{1-M} \frac{1}{R}$ \\
\hline boost & $\frac{2 V}{R} \sqrt{\frac{M}{K(M-1)}}$ & $\frac{M-1}{M^{3}} R$ & $\frac{M}{M-1} \frac{1}{R}$ & $\frac{2 V}{R \sqrt{K M(M-1)}}$ & $\frac{M-1}{M} R$ & $\frac{M(2 M-1)}{M-1} \frac{1}{R}$ \\
\hline $\begin{array}{l}\text { buck- } \\
\text { boost }\end{array}$ & $\frac{2|V|}{R \sqrt{K}}$ & $\frac{R}{M^{2}}$ & 0 & $\frac{2|V|}{R \sqrt{K} M}$ & $R$ & $\frac{2 M}{R}$ \\
\hline
\end{tabular}

TABLE III. Definition of the elements in the canonical circuit model of Fig. 22 for the three common converters of Fig. 1 operating in the discontinuous conduction mode.

\begin{tabular}{|c|c|c|c|}
\hline type & $G_{\text {og }}$ & God & $w_{p}$ \\
\hline buck & $M$ & $\frac{2 V(1-M)^{3 / 2}}{\sqrt{K} M(2-M)}$ & $\frac{2-M}{1-M} \frac{1}{R C}$ \\
\hline boost & $M$ & $\frac{2 V}{2 M-1} \sqrt{\frac{K M}{M-1}}$ & $\frac{2 M-1}{M-1} \frac{1}{R C}$ \\
\hline $\begin{array}{c}\text { buck } \\
\text { boost }\end{array}$ & $M$ & $\frac{V}{\sqrt{K} M}$ & $\frac{2}{R C}$ \\
\hline$G_{v g}=\frac{\hat{v}}{\hat{v}_{g}}=G_{o g} \frac{1}{1+s / w_{p}}$ & $; G_{v d}=\frac{\hat{v}}{\hat{d}}=G o d \frac{1}{1+S / w_{p}^{2}}$ \\
\hline
\end{tabular}

TABLE IV. Summary of the ac transfer properties of the three common converters of Fig. 1 operating in the discontinuous conduction mode.

All the results presented in this section are applicable only to the discontinuous conduction mode of operation of these three switching converters. To determine when these results ought to be applied and when those for continuous conduction mode [2], the boundary between the two modes of operation is determined for these three converters and tabulated in the next section.

\subsection{Determination of the boundary between two conduction modes}

As explained in detall in Section 3.1 the criteria for determination of the converter conduction mode are 
boundary between the two conduction modes

$$
K=K_{\text {crit }} \quad \text { or } \quad R=R_{\text {crit }}
$$

continuous conduction mode

$$
\mathrm{K}>\mathrm{K}_{\text {crit }} \text { or } \mathrm{R}<\mathrm{R}_{\text {crit }}
$$

discontinuous conduction mode

$$
\mathrm{K}<\mathrm{K}_{\text {crit }} \text { or } \mathrm{R}>\mathrm{R}_{\text {crit }}
$$

where $K$ is as defined before $K=2 L / R T=2 L f / R$. Following the same procedure outlined In Section 3.1 for the boost converter example, the parameters $K$ and $R$ can easily be found for the other two converters and all resutls are shown tabulated in Table $V$.

\begin{tabular}{|c|c|c|c|c|}
\hline \multirow{2}{*}{$\begin{array}{c}\text { converter } \\
\text { type }\end{array}$} & \multicolumn{2}{|c|}{ open-locf consideration } & \multicolumn{2}{c|}{ closed - loop consideration } \\
\cline { 2 - 5 } buck & $1-D$ & $\frac{R_{\text {nom }}}{1-D}$ & $1-M$ & $\frac{R_{\text {nom }}}{1-M}$ \\
\hline bocit $(D)$ & $R_{\text {crit }}\left(D, R_{\text {nom }}\right.$ & $K_{\text {crit }}(M)$ & $R_{\text {crit }}\left(M, R_{\text {nom }}\right)$ \\
\hline $\begin{array}{c}\text { buck- } \\
\text { boost }\end{array}$ & $D(1-D)^{2}$ & $\frac{R_{\text {nom }}}{D(1-D)^{2}}$ & $\frac{M-1}{M^{3}}$ & $\frac{M^{3}}{M-1} R_{\text {nom }}$ \\
\hline
\end{tabular}

TABLE V. Determination of the boundary between the two conduction modes, expressed for open-loop as well as for closed-loop considerations.

In Table $V$ nominal resistance $R_{\text {nom }}$ is a design parameter defined by

$$
R_{\text {nom }}=2 L f_{s}
$$

It has already been demonstrated in Section 3.1 for the boost converter that parameter $K$ can be chosen $(K>4 / 27)$, such that the converter is always operating in the continuous conduction mode regardless of the operating point, that is dc duty ratio $D$, while the discontinuous conduction mode can occur only for $K<4 / 27$, and then only for a portion of the dynamic range of duty ratio $D$. The same holds true for the other two converters, and the following criteria can be set:

a) when $K>K_{M}$ the converter is always in continuous conduction mode regardless of $D$.

b) when $\mathrm{K}<\mathrm{K}_{\mathrm{M}}$ discontinuous conduction mode can occur, but only for a limited range of duty ratio $D$.

Parameter $K_{M}$ is actually the maximum of the duty ratio $D$ dependent function of first column in Table $V$, and is for comparison purposes listed in Table VI.

\begin{tabular}{|c|c|c|c|}
\hline & buck & boost & $\begin{array}{c}\text { buck } \\
\text { boost }\end{array}$ \\
\hline$K_{M}$ & 1 & $\frac{4}{27}$ & 1 \\
\hline
\end{tabular}

TABLE VI. Summary of the parameter $K_{M}$ determining the region of unconditional continuous conduction for three common converters of Fig. 1.

From Table VI it is obvious that when $K>1$ any of the three converters 1 isted will always operate in the continuous conduction mode, and when $K<4 / 27$ each of them will operate in the discontinuous conduction mode for a portion of the duty ratio range. With this, and the first column in Table II, the de voltage gain as a function of duty ratio can be shown as in F1g. 23b for $K<4 / 27$, while the corresponding result for continuous conduction mode is illustrated for comparison purposes in Fig. 23a for $K>1$.

a) continuous conduction b) discontinuous conduction
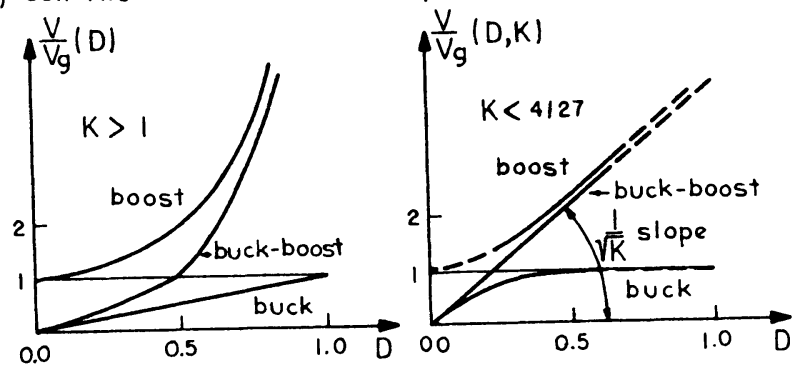

Fig. 23. Comparison of the dc voltage gain characteristics in the two conduction modes for the common converters of Fig. 1.

In F1g. 23b heavy lines designate the region of actual discontinuous conduction operation, whereas dotted lines signify that the continuous conduction mode takes over and the dc gain characteristics begin to follow those for the continuous conduction mode (see for comparison Fig. 8). From Fig. 23b it 18 also evident that in the buck and the buck-boost converter, the transition between the two conduction modes occurs only once at higher duty ratio $\mathrm{D}$, and not also at the lower end as it does in the boost converter. Therefore during inftial start-up of the converter, when the duty ratio changes from zero to the value required by the steady-state gain $M$, the two converters (buck and buck-boost) can be designed to stay in the discontinuous conduction mode only, even in this transitional period.

We now present another viewpoint, which in an interesting pictorial way and a unique frequency interpretation, 1lluminates the determination of the converter operating mode and the basic small switching ripple requirement. Namely, from Fig. 1 it is apparent that the three common converters essentially consist of the single switch $S$ positioned differently among the source voltage $V_{f}$ and three elements, Inductance $L$, capacitance $C$, and Poad R. With only these three elements three different "Inherent" frequencles can be defined regardless of the converter type. Two of them, $\omega_{\alpha}$ and $\omega_{c}$, termed natural frequencles, are defined as

$$
\omega_{\alpha}=\frac{1}{2 \mathrm{RC}}, \quad \omega_{c}=\frac{1}{\sqrt{L C}}
$$

However, yet another "Inherent" frequency $\omega_{\beta}$ can be defined by these three elements as

$$
\omega_{\beta}=\frac{R}{2 L}
$$

The dimensionless parameter $K$, which plays a crucial role in the determination of the conduction mode, can now be expressed as

$$
K=\frac{f_{s}}{w_{B}}
$$

Therefore, the position of this new frequency $\omega_{8}$ with respect ot the switching frequency $f_{s}$ determines the conduction mode. Hence for $\boldsymbol{K}>1$ or $\omega_{\beta}<f$, each of the three converters will always be in continuous conduction mode regardless of $D$. On the other hand, $\omega_{q} \ll f_{s}$ and $\omega_{c} \ll f_{\text {f }}$ are requirements for small switching ripple. The information contained in the position of these three "inherent" frequencies $\omega_{\alpha}, \omega_{\beta}$ and $f$ with respect to the switching frequency $f^{\alpha}$ is ${ }^{\beta}$ concisely summarized in Fig. 24. The diagram in Fig. 24, with the 


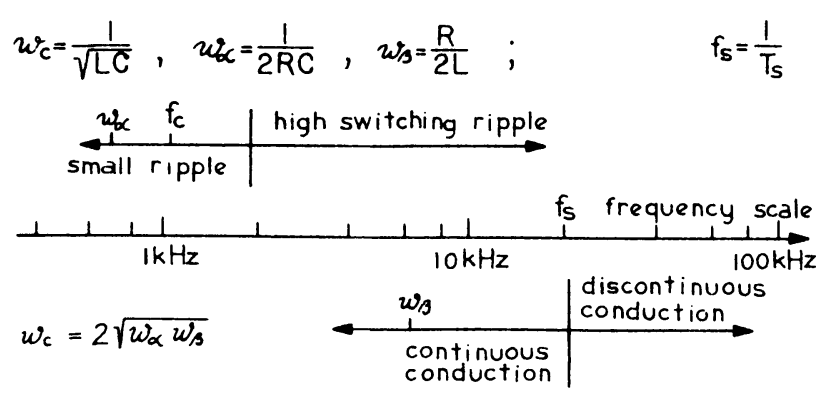

Fig. 24. Frequency interpretation of the conduction mode type and small switching ripple requirement.

help of definitions (92) and (93), displays in a convincing manner the interplay between conduction mode types, switching ripple requirement and choice of parameter values $L, C, R$, and $f$. For example, increase of load $R$ can cause change to fiscontinuous conduction mode without deterioration in switching ripple. However, if inductance $L$ or switching frequency is reduced, change to discontinuous conduction mode can occur, but at the price of higher switching ripple since separation between $\omega_{\text {and }} \hat{i}_{\text {is }}$ is also reduced. One would have to increase capacitance $C$ to remain at an acceptable switching ripple level. Thus the frequency diagram of Fig. 24 gives valuable insight, both qualitative and quantitative, into the basic relationships inherent to switching converters. It 18 interesting that from (92) and (93) a very simple relationship follows

$$
\omega_{c}=2 \sqrt{\omega_{\alpha} \omega_{B}}
$$

which may further facilitate quantitative analysis.

\subsection{Experimental verification of the transfer properties}

Both dc and ac transfer properties have been experimentally verified on a circuit breadboard of the buckboost converter shown in Fig. 12c. Because of lack of space, only cursory experimental verification is included here.

The buck-boost converter was chosen because of several unique features which clearly distinguish it form the other two converters, and which are easy to check. A quick look at Table II, for example, reveals that it is the only converter whose second interval $D_{2} T$ is independent of the operating conditions (duty ratio $D$ or gain $M$ ), but rather is fixed determined by the parameter $\mathrm{K}$ only.

Likewise, a look at Table III shows that the ac resistance $r_{2}$ is also independent of steady-state operating condition (gain $M$ ). Therefore, the single pole of the two transfer funcitons $G$ and $G$ does not move with change of operating condit $\mathrm{Y}_{\mathrm{o}}$ (gain $\mathrm{M}$ ) as it does in the other two converters.

Finally, the open-1oop input impedance of the buckboost converter is $R_{f}=R / M^{2}$ since there is no internal feedback $\left(g_{1}=0\right)$. Hence the input impedance is purely resistive, which is not the case for the other two converters.

The transfer properties have been verifled on the test buck-boost converter with the following switching components: transistor $2 \mathrm{~N} 2880$ and diode TRW SVD 100-6.

Dc gain measurements

For the choice of element values $\mathrm{L}=890 \mu \mathrm{H}, \mathrm{C}=12 \mu \mathrm{F}$, $R=220 \Omega, f_{s}=10 \mathrm{kHz}$ and $V_{g}=6 \mathrm{~V}$ we compute $\mathrm{K}=2 \mathrm{Lf} / \mathrm{s}=$ 0.81 and $D_{2}=\sqrt{K}=0.28$. Therefore, the buck-boost con- verter operates in the discontinuous conduction mode from $D=0$ until $D=1-D_{2}=0.72$, and the experimental dc gain characteristic is shown in this duty ratio range on Fig. 25.

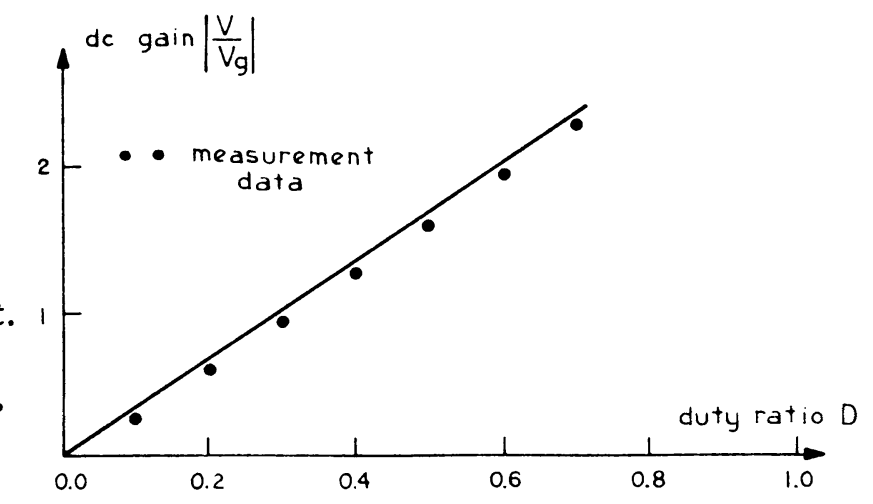

Fig. 25. Dc voltage gain measurements for the buck-boost converter in the discontinuous conduction mode.

As seen in Fig. 25, the experimental points follow very closely the theoretical straight line characteristic. The experimental data, however, are slightly lower than the theoretical curve since the transistor saturation voltage and diode drop have not been accounted for in the theoretical model, although this could easily be accomplished. The inductor current waveform was monitored, and confirmed discontinuous conduction operation for $D E[0,0.72]$ while $D_{2}$ measured was constant as predicted at $\mathrm{D}_{2}=0.28$.

Ac transfer function measurements

The duty ratio modulation $\hat{\mathrm{d}}$ to output voltage $\hat{\mathrm{v}}$ transfer function $G$ is now measured using the describing function measurement technique [11] and results are shown in $\mathrm{Fig} .26$.

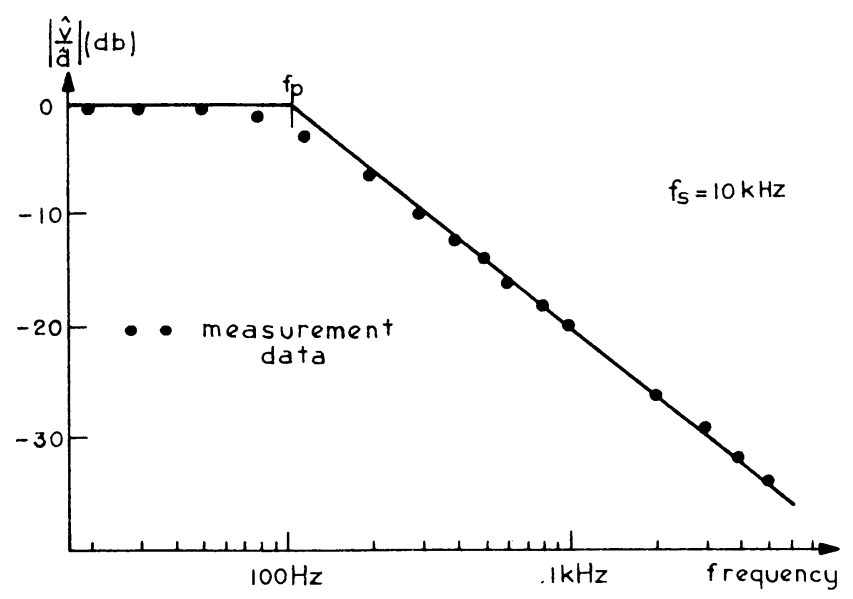

Fig. 26. Experimental magnitude-frequency response of $G_{1}=\hat{v} / \hat{d}$ transfer function for buck-boost converter in the discontinuous conduction mode.

The element values used are the same as for the dc measurements, except that the inductance was increased four times to $L=3.5 \mathrm{mH}$ to reduce the superimposed switching ripple and to reduce the ringing effect in the $\mathrm{D}_{3} \mathrm{~T}$ interval. Hence for $\mathrm{L}=3.5 \mathrm{mH}, \mathrm{C}=12 \mu \mathrm{F}, \mathrm{R}=220 \Omega$, $\mathrm{f}_{\mathrm{s}}^{3} \stackrel{\mathrm{s}}{\mathrm{s}} 10 \mathrm{kHz}, \mathrm{V}_{\mathrm{f}}=6 \mathrm{~V}$ we calculate $\mathrm{K}=1.62$ and $\mathrm{D}_{2}=0.56$. The range of aiscontinuous conduction operation ${ }^{2}$ is then reduced to $D \in[0,0.44]$. The single pole of the transfer functions $G_{\text {g }}$ and $G$ (see Table IV) becomes $f=1 / \pi R C=$ $120 \mathrm{~Hz}$, which ${ }^{1 / 5}$ in excellent agreement with the experimental data shown in Fig. 26. 
The measurements were repeated for several operating points in the discontinuous conduction region, namely, for $D=0.1,0.2,0.3$, and 0.4 but the single pole at $f_{p}$, as predicted, did not move.

The experimental measurements therefore have confirmed the high degree of accuracy of the canonical circuit model (Fig. 22) for the discontinuous conduction mode of operation.

The question of input properties of switching converters and regulators, and particularly of openand closed-1oop input impedances, is thoroughly analyzed in the next section on modelling of a switching mode regulator in the discontinuous conduction mode.

7 MODELLING OF SWITCHING REGULATOR IN DISCONTINUOUS CONDUCTION MODE

This section demonstrates how the canonical circuit model for a switching converter operating in the discontinuous conduction mode can easily be incorporated into the complete switching-mode regulator model. Consider now a switching-mode regulator as shown in Fig. 27, an illustrative example since the discussion applies to any converter.

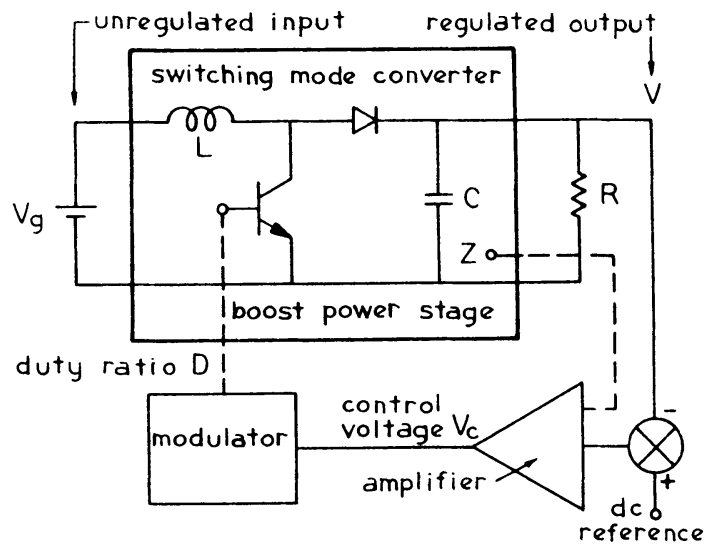

Fig. 27. Switching-mode regulator

\subsection{Modulator stage modelling and complete regulator circuit mode1}

So far, we have obtained the canonical circuit model for the switching-mode converter. The next step in development of the regulator equivalent circuit is to obtain a model for the modulator. This is easily done by writing an expression for the essential function of the modulator, which is to convert an (analog) control voltage $v$ to the switch duty ratio $D$. This expression can be written $D=V / V$ in which, by definition, $V$ is the range of control ${ }^{c} 1_{\mathrm{gnal}}^{\mathrm{m}}$ required to sweep the duty ratio over its full range from 0 to 1 . A small variation $\hat{v}_{\text {c }}$ superimposed upon $v_{c}$ therefore produces a corresponding văriation $\hat{d}=\hat{v}_{c} / v_{\text {in }}{ }^{C} D$, which can be generalized to account for a nonuniform frequency response as

$$
\hat{\mathbf{d}}=\frac{\mathbf{f}_{m}(s)}{v_{m}} \hat{v}_{c}
$$

in which $f_{f}(0)=1$. Thus, the control voltage to duty ratio small-signal transmission characteristic of the modulator can be represented in general by the two parameters $V_{m}$ and $f_{1}(8)$, regardless of the detalled mechanism by which the modulation is achieved.
The inclusion of the canonical circuit model (Fig. 22 ) and an appropriate model for the modulator stage (96) into the switching regulator (Fig. 27) results in a complete circuit model of a switching regulator in the discontinuous conduction mode, as shown in Fig. 28.

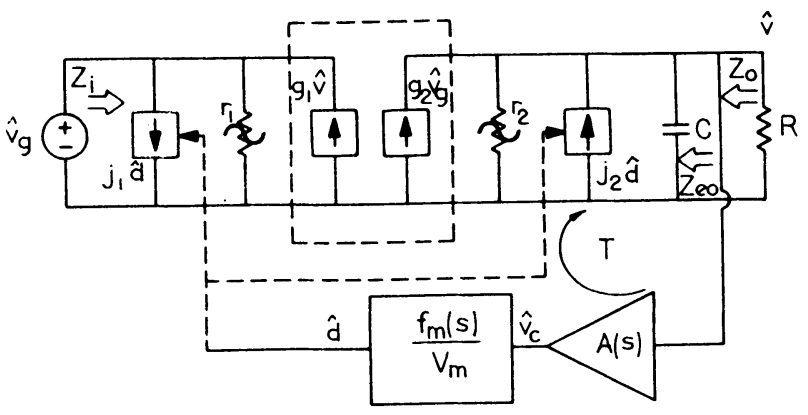

Fig. 28. General ac small-signal circuit model for the suitching regulator of Fig. 25 operating in the discontinuous conduction mode.

The generator symbol for the current generators $f_{1}(s) \hat{d}$ and $f_{2}(s) \hat{d}$ at the input and output ports, respectfvely, has been changed from a circle to a square to emphasize that in the closed-loop regulator they have become dependent generators (on output voltage varlation $v$ in particular). A closer look at the circuit model in Fig. 28 reveals some unique properties of this negative feedback circuit. Namely, it has been previously shown in Section 3 that only the output port network (consisting of current generators $g_{2} \hat{v}_{j}, j_{2} \hat{d}$, resistances $r_{2}$ and $R$ and capacitance C) effectively takes part in determination of the open-loop transfer functions $G$ and $G$. The Immediate implication of this is that for Ylleal source voltage $v_{g}$, the loop gain $T$ is defined only with respect to the oulput port as shown in Fig.28. Likewise, the output impedance $Z$ and line transmission characteristic F (audio-susceptiblilty) become solely defined in terms of the output port elements, while the input port takes part only in determination of the input impedance $z$. This is easily confirmed by analysis of the equivalent circuit in Fig. 28 , which leads to

$$
\begin{aligned}
T & =G_{v d}(s) A(s) f_{m}(s) / V_{m} \\
Z_{0} & =\frac{Z_{e o}(s)}{1+T} \\
F & =\frac{G_{v g}(s)}{1+T} \\
\frac{1}{Z_{i}} & =-\frac{T}{1+T}\left(\frac{G g}{G_{v d}} j_{1}-\frac{1}{r_{1}}\right)+\frac{1}{1+T}\left(\frac{1}{r_{1}}-g_{1} G_{v g}\right)
\end{aligned}
$$

The first three expressions are rather obvious and are a consequence of the general results of linear feedback theory. They also confirm that $T, Z$, and $F$ are functions of the output port elements only', since the open-loop transfer functions $G_{v g}$ and $G_{v d}$ are independent
of Input port elements.

It should be noted, however, that this peculfar dependence of some feedback quantities $T, Z$, and $F$ on output port elements only, is a quite special case, which is a consequence of the ideal source voltage $\hat{v}_{\text {. }}$. If the source voltage had an internal impedance, or $\mathrm{g}^{\circ}$ input filter were included in front of the converter, even the open-loop transfer functions $G$ and $G$ would become dependent on all circuit elements, the feedback quantities even more so, and this special feature would 
disappear. This once again demonstrates how powerful these converter equivalent circuit models are, since any of such additional effects can be directly included in the circuit model of Fig. 28, owing to its complete circuit representation of the converter properties.

We now Investigate in more detail the important input properties of the circuit model in Fig. 28.

\subsection{Input properties of switching regulators in discontinuous conduction mode}

As seen in (100) the input impedance $z$ is also dependent on the input quantities $f_{1}, r_{1}$, and $g_{1}$. In addition the input duty ratio dependent current generator $f_{1}$ is now responsible for the negative input impedance at low frequencles. Indeed, if $f_{1}=0$, and since at low frequencies $T \rightarrow \infty$, the input resistance $R$ would appear to be positive, in obvious conflict with the actual physical requirement.

Let us now verify this for the discontinuous conduction mode, and consider first the limiting case of (100) for high loop gain $\mathrm{T} \rightarrow \infty$ (at low frequencles)

$$
\frac{1}{R_{1}}=-\left(\frac{G v_{g}}{G_{v d}} j_{1}-\frac{1}{r_{1}}\right)
$$

From the circuit model in Fig. 28 the converter open-1oop transfer functions $G_{v g}$ and $G_{v d}$ are easily found $a s$

$$
\begin{aligned}
& G_{v g}=g_{2}\left(r_{2} \| R\right) \frac{1}{1+s C\left(r_{2} \| R\right)} \\
& G_{v d}=j_{2}\left(r_{2} \| R\right) \frac{1}{1+8 C\left(r_{2} \| R\right)}
\end{aligned}
$$

By use of ( 102$)$ in (101) we finally obtain the closed-loop incremental resistance $R_{1}$ as

$$
R_{1}=-\left(\frac{J_{1}}{J_{2}} g_{2}-\frac{1}{r_{1}}\right)
$$

Using now the definitions of element values $j_{1}, j_{2}$, $\dot{g}_{2}$, and $r_{1}$ from Table III in (103), we obtain for del three converters (buck, boost and buck-boost) that

$$
R_{1}=-\frac{R}{M^{2}}=-\left(\frac{v_{8}}{v}\right)^{2} R
$$

From (103) it is also evident that despite the presence of the positive term, the negative term has prevailed, correctly predicting the negative closed-loop input resistance.

Let us now consider the other extreme when the loop gain is very small, that is $\mathrm{T} \rightarrow 0$ (or equivalently at high frequencies). Then, the input impedance approaches the open-10op input impedance $Z_{\text {in }}$ obtained from $(100)$ as

$$
\frac{1}{z_{\text {In }}}=\frac{1}{r_{1}}-8_{1} G \mathrm{vg}
$$

The same result could be obtained directly from the openloop converter model in Fig. 22. From (105) it seems as though $Z_{\text {f }}$ could be negative owing to this negative internal effect of the current generator $g_{1} v$ in the model of Fig. 22. However, this is not true, since the lowfrequency value of the open-loop input impedance $R_{\text {in }}$ becomes from (105)

$$
R_{\text {in }}=\frac{r_{1}}{1-g_{1} r_{1} g_{2}\left(r_{2} \| R\right)}
$$

Again by using element definitions from Table III in (106) we get for all three converters

$$
R_{\text {in }}=\frac{R}{M^{2}}=\left(\frac{v_{g}}{v}\right)^{2} R
$$

which correctly predicts the open-loop low-frequency input resistance to be positive.

From these results and the corresponding one for continuous conduction mode [1], it follows that the closedloop low-frequency input resistance $R$ is given by (104) regardless of the conduction mode type and switching converter type (buck, boost, or buck-boost). The same is also true for the open-loop low-frequency input resistance $R_{\text {in }}$ given by (107).

Hence, this section has confirmed that the canonical circuit model for discontinuous conduction mode (F1g. 28) properly models the regulator input properties (closedloop input impedance) in much the same way as the canonical circuit model for continuous conduction mode $[1,2]$ did, through the presence of duty ratio dependent current generators at the input of the converter model. The immediate consequence of this is that the regulator c1rcuit model (F1g. 28) is a complete c1rcuit model which represents all essential properties; input, output and transfer properties.

\section{$8 \quad$ CONCLUSIONS}

A general method for modelling any three-state switching converter operating in the discontinuous conduction mode has been presented. The fundamental step is in replacement of the state-space descriptions of the three switched networks (3) by their average ( 8 ) over the single period $T$, the same step as taken for any ordinary three-state converter. This is then supplemented by additional constraints $(9)$ and (10) which properly account for the discontinuous conduction mode of operation.

The subsequent perturbation and 11nearization steps are applied not only to the state-space or circult averaged models but also to the constraints, which then provide the additional information needed to define completely both dc and ac smal1-signal models.

An extensive analysis of the dc conditions in the discontinuous conduction mode has been given, in Section 3 , which then enabled the definition of the boundary between the two operating modes for a specific boost converter example. An easily interpretable formula ((45) or (49)) led to simple criteria ((46), (47) and (48)) for determination of the converter. mode of operation.

Analysis of the dynamic (ac small-signal) model confirmed the general modelling prediction - reduction of the system order by one. Thus, common converters of F1g. 1 showed a single-pole frequency response in the discontinuous conduction mode, as apposed to their twopole response in the continuous conduction mode.

Then, following the hybrid modelling path (Section 4) and the circuit averaging path (Section 5), a new c1rcult model (F1g. 18) with a rather unusual topological structure is obtained for the boost converter, which provides a complete model for dynamic (ac smallsignal) behavior.

The canonical circuit model with the same topology (F1g. 18), but with different element values, is obtained in Section 6 for the other two converters of Fig. 1 , and the results are conventently summarized in various tables. Experimental verification of dc and ac transfer properties of a buck-boost converter in discontinuous conduction mode are also provided. 
Finally, the model of the switching-mode regulator operating in the discontinuous conduction mode is obtained in Section 7, and important input properties (both open- and closed-1oop) are thoroughly analyzed.

The outlined method is general and directly applicable to investigation of the discontinuous conduction mode in more complex switching converter structures, such as those described in $[12,13]$, involving more than a single inductor.

\section{REFERENCES}

[1] Slobodan Cuk, "Modelling, Analys1s, and Design of Switching Converters," Ph.D. thesis, California Institute of Technology, November 1976.

[2] R. D. Middlebrook and S. Cuk, "A General Unified Approach to Modelling Switching-Converter Power Stages," IEEE Power Electronics Specialists Conference, 1976 Record, pp. 18-34, (IEEE Publication 76 CHO 1084-3AES). (Also to be published in International Journal of Electronics, London).

[3] R. D. Middlebrook and S. Ćuk, "Modelling and Analysis Methods for Dc-to-Dc Switching Converters," IEEE International Semiconductor Power Converter Conference, 1977 Record, pp. 90-111 (IEEE Pub1ication $77 \mathrm{CH}$ 1183-31A).

[4] R. Prajoux, J. C. Marpinard, and J. Jalade, "Accurate Mathematical Modelling of PWM Power Regulators," Translation of Report, Laboratoire d'Automatique et d'Analyse des Systems, C.N.R.S., issued under ESTEC Contract No. 2026 AK, May 74, 25 pp.

[5] A. Capel, J. G. Ferrante, and R. Prajoux, "Stability Analysis of PWM Controlled DC/DC Regulator with DC and AC Feedback Loops," IEEE Power Electronics Specialists Conference, 1974 Record, pp. 246-254, (IEEE Publication 74 CHO 863-1-AES).

[6] A. Capel, J. G. Ferrante, and R. Prajoux, "State Varlable Stability Analysis of Multiloop PWM Controlled Regulators in Light and Heavy Mode," IEEE Power Electronics Specialists Conference, 1975 Record, pp. 91-102 (IEEE Publication 75 CHO 965-4-AES).

[7] H. A. Owen, A. Capel, and J. G. Ferrante, "S1mulation and Analysis Methods for Sampled Puwer Electronic Systems," IEEE Power Electronics Speclalists Conference, 1976 Record, pp. 45-55 (IEEE Publication $76 \mathrm{CH}$ 1084-AES).

[8] F. C. Lee, Y. Yu, and J. E. Triner, "Modelling of Switching Regulator Power Stages with and without Zero-Inductor Current Dwel1 Time," IEEE Power Electronics Specialists Conference, 1976 Record, pp. 62-72, (IEEE Publication $76 \mathrm{CH} \mathrm{1084-3AES).}$

[9] A. Cape1, J. G. Ferrante, and R. Prajoux, "Dynamic Behavior and $Z$ Transform Stability Analysis for DC/DC Regulators with a Nonlinear PWM Controlled LOop," IEEE Power Electronics Speclalists Conference, 1973 Record, pp. 149-157, (IEEE Publication CHO 787-2AES).

[10] M. Clique and A. J. Fossard, "Development et App11cations de Modeles Numeriques pour 1'Evaluation des Systems de Puissance," ler Rapport d'Advancement, April 1976, Contract ESTEC no. 2590/75AK.

[11] R. D. Middlebrook, "Measurement of Loop Gain in Feedback Systems," International Journal of Electronics, 1975, vol. 38, no. 4, pp. 485-512.
[12] Slobodan Cuk and R. D. Middlebrook, "A New Optimum Topology Switching Dc-to-Dc Converter," IEEE Power Electronics Speciallsts Conference, Palo Alto, Calif., June 14-16, 1977.

[13] Slobodan Cuk and R. D. Middlebrook, "Coupled-Inductor and Other Extensions of a New Optimum Topology Switching Dc-to-Dc Converter, "IEEE Industry Applications Society Annual Meeting, Los Angeles, Calif., Oct. 2-6, 1977.

\section{APPENDIX A}

STATE-SPACE AVERAGING STEP EXTENDED TO CONVERTERS WITH MULTISTRUCTURAL (THREE OR MORE) TOPOLOGICAL CHANGES

We derive the state-space averaging step for switching converters characterized by three structural changes within each switching period. Each topological structure can be described as before by linear statespace equations, hence

$\dot{x}_{1}=A_{1} x_{1}+b_{1} v_{g} \quad$ for interval $d_{1} T_{8}, \quad\left(0 \leq t \leq t_{1}\right)$

$\dot{x}_{2}=A_{2} x_{2}+b_{2} v_{g}$ for interval $d_{2} T_{8}, \quad \cdot\left(t_{1} \leq t \leq t_{2}\right)$

$\dot{x}_{3}=A_{3} x_{3}+b_{3} v_{g}$ for interval $d_{3} T_{8}, \quad\left(t_{2} \leq t \leq T_{8}\right)$

Two boundary conditions are now imposed. Since the state-space vector is continuous in transition from first to second and from second to third regions,

$$
\begin{aligned}
& x_{2}\left(t_{1}\right)=x_{1}\left(t_{1}\right) \\
& x_{3}\left(t_{2}\right)=x_{2}\left(t_{2}\right)
\end{aligned}
$$

Solution of (A.1) under the small signal assumption for $\hat{v}_{g}$ (where $v_{g}=v_{g}+\hat{v}_{g}$ and $\hat{v}_{g} \ll v_{g}$ ) ylelds

$$
\begin{aligned}
& x_{1}(t)=e^{A_{1} t} x_{1}(0)+v_{g} B_{1}(t) b_{1} \quad \text { for } t \in\left[0, t_{1}\right] \\
& x_{2}(t)=e^{A_{2}\left(t-t_{1}\right)} x_{2}\left(t_{1}\right)+v_{g} B_{2}\left(t-t_{1}\right) b_{2} \text { for } t \varepsilon\left[t_{1}, t_{2}\right] \\
& x_{3}(t)=e^{A_{3}\left(t-t_{2}\right)} x_{2}\left(t_{2}\right)+v_{8} B_{3}\left(t-t_{2}\right) b_{3} \text { for } t \varepsilon\left[t_{2}, T_{8}\right]
\end{aligned}
$$

where

$$
B_{1}(t)=\int_{0}^{t} e^{A_{1} \tau} d \tau, \quad 1=1,2,3
$$

Use of boundary conditions (A.2) in (A.3) gives

$$
\begin{aligned}
& x_{3}\left(T_{s}\right)=e^{A_{3} d_{3} T_{s}} e^{A_{2} d_{2} T_{s} e^{A_{1} d_{1} T} s_{s}} x_{1}(0)+ \\
& +v_{g}\left[e^{A_{3} d_{3} T_{s}} e^{A_{2} d_{2} T_{s_{B}}}{ }_{B_{1}}\left(d_{1} T_{s}\right) b_{1}+\right. \\
& \left.+e^{A_{3} d_{3} T} s_{B_{2}}\left(d_{2} T_{s}\right) b_{2}+B_{3}\left(d_{3} T_{s}\right) b_{3}\right]
\end{aligned}
$$

With introduction of the linear approximations

$$
e^{A_{1} d_{1} T_{s}} \sim I+A_{1} d_{1} T_{s}, \quad 1=1,2,3
$$


into (A.4) and (A.5), and after retention of only firstorder terms (1inear in $T_{s}$ ), (A.5) reduces to

$$
x_{3}\left(T_{s}\right)=\left(I+d_{1} A_{1}+d_{2} A_{2}+d_{3} A_{3}\right) x_{1}(0)+\left(d_{1} b_{1}+d_{2} b_{2}+d_{3} b_{3}\right) v_{g}
$$

This leads to a single continuous linear system

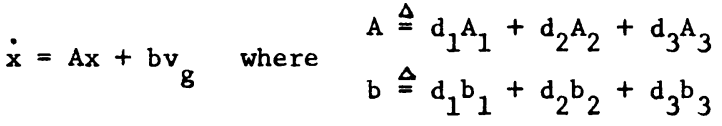

It remains, finally, to characterize the statespace averaging step for the generalized switching converter with $n$ structural changes within each switching period, namely, one described by

$$
\dot{x}=A_{1} x+b_{1} v_{g}, \quad \begin{aligned}
& d_{1} T_{s}=t_{1}-t_{1-1} \\
& t \in\left[t_{1-1}, t_{j}\right]
\end{aligned} \quad 1=1,2, \ldots, n
$$

for which the corresponding basic state-space averaged model is

$$
\begin{aligned}
\dot{x}=\mathrm{Ax}+\mathrm{bv} \mathrm{g}_{\mathrm{g}} ; & =\sum_{i=1}^{n} \mathrm{~d}_{1} \mathrm{~A}_{1} \\
\mathrm{~b} & =\sum_{1=1}^{n} \mathrm{~d}_{1} \mathrm{~b}_{1}
\end{aligned}
$$

As an illustration of a switching converter with such multistructural change, consider the converter shown in Fig. A.la whose two switches $\mathrm{S}_{1}$ and $\mathrm{S}_{2}$ are driven as specifled in F1g. A,1b. The two switches $\mathrm{S}_{1}$ and $S_{2}$ are shown in their "on" position in Fig. A.la. It can easily be recognized that this converter is actually a boost converter cascaded by a buck converter whose switches are driven synchronously but with different duty ratios, $d_{1}$ and $d_{1}+d_{2}$ respectively.

a)

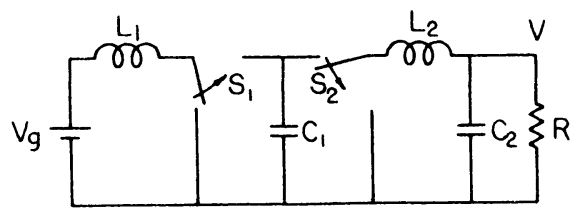

b)

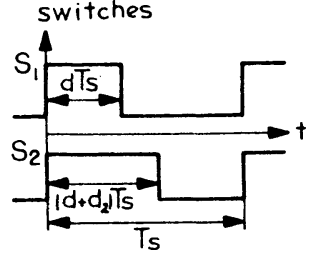

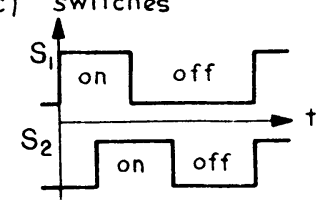

c) switches

Fig. A.1 switching converter exhibiting multistructural change: a) boost converter cascaded by a buck converter; b) switch drive for "three-state" behavior; c) switch drive for "four-state" behavior.

However, if this converter is looked upon as single system, the switching action of $\mathrm{Fig}$. A.lb would produce periodic sequential change among three different structures (shown in Fig. A.2b, c, and d), while that of Fig. A.1.c would produce periodic sequential change among all four different switched networks of Fig. A.2. In any case, it demonstrates the feasibility of realization of a switching converter having three or more switched network configurations, even in the continuous conduction mode of operation. a) $S_{1}$ on, $S_{2}$ off :

b) $S_{1}$ on, $S_{2}$ on:

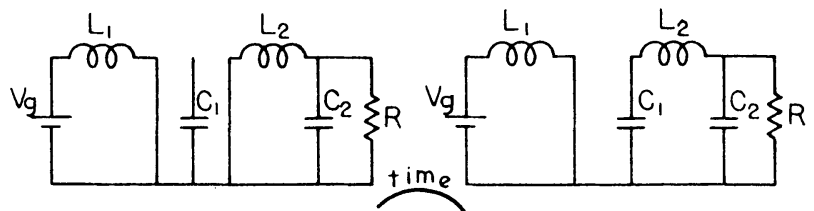

d) $S_{1}$ off, $S_{2}$ off:

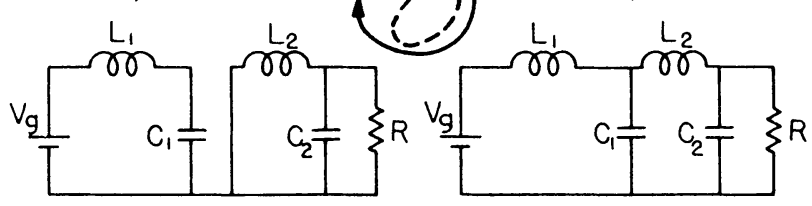

Fig. A.2 Various switched networks for the converter in Fig. A. 1a.

On the other hand if the converter is looked upon as consisting of cascaded boost and buck converters and each of them has been modelled separately as a "two-state" converter as in [2], and their models put together, the same result would have been obtained.

However, for the discontinuous conduction mode, In addition to the state-space averaging step (A.8) for "three-state" converters, other restrictions $((1),(2))$ are imposed to reflect the limited behavior of inductor current ( $F I_{g}$. 2b) with fixed (zero) boundary values.

But in any case, for either continuous conduction mode [2], or discontinuous conduction mode, the corresponding state-space averaging step is justified on the basis of the fundamental performance requirement for switching dc-to-dc converters of small (negligible) switching ripple, as follows:

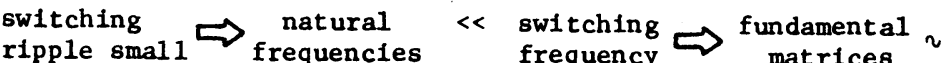

$\sim \underset{\text { approximation }}{\text { linear }} \underset{\text { averaging step }}{\text { state-space }}$

This, together with proper inclusion of the inductor current discontinuity as additional constraints (1), (2), enable the extremely simple, powerful and accurate scheme for modelling and analysis of switching converters in discontinuous conduction mode to be established. 